BULLETIN OF THE

AMERICAN MATHEMATICAL SOCIETY

Volume 77, Number 3, May 1971

\title{
THE FACTORIZATION PROBLEM FOR NONNEGATIVE OPERATOR VALUED FUNCTIONS
}

\author{
BY MARVIN ROSENBLUM AND JAMES ROVNYAK ${ }^{1}$
}

Introduction. Let $F$ be a function defined on the circle $\Gamma$ $=\left\{e^{i \theta}: 0 \leqq \theta<2 \pi\right\}$ or line $R=(-\infty, \infty)$ whose values are nonnegative operators on a separable complex Hilbert space. We are concerned with the problem of finding conditions that $F=G^{*} G$ a.e. where $G$ is the strong boundary value function of a suitable operator valued analytic function defined in the disk $|z|<1$ or half-plane $y>0$. Mainly we are interested in special classes of functions in which such a factorization is always possible.

Our study is motivated by the Fejér-Riesz theorem on the factorization of nonnegative trigonometric polynomials, and Ahiezer's version [1] of its generalization to entire functions of exponential type which are nonnegative on the real axis. Both results generalize to operator valued functions, and, in fact, both appear as special cases of a very general result (Theorem 3.1).

More generally we present a unified treatment of the factorization problem, and thus much of $\S 1$ is expository. There we develop the theory of a corresponding abstract factorization problem for nonnegative Hilbert space operators. Both the results and methods of $\S 1$ are purely operator theoretic. In $\$ 2$ we show how the abstract theory relates to the theory of operator valued functions defined on the circle $\Gamma$ or line $R$. The main applications to the factorization problem for nonnegative operator valued functions are deferred to $\$ 3$.

The factorization problem arises in the prediction theory of stationary stochastic processes. For this connection see Helson and Lowdenslager [11], Rozanov [27], and Wiener and Masani [28].

We wish to thank Professor Loren Pitt for calling our attention to the paper by E. Robinson [23]. We have extended Robinson's results in $\S 2$.

\footnotetext{
${ }^{1}$ Based on an invited address by the first author at the meeting of the Society in Baton Rouge, Louisiana, November 21, 1969. Also presented by the second author at the Symposium on Operator Theory, Indiana University, June 3, 1970. Research supported by NSF Grant GP 19852.

AMS 1970 subject classifications. Primary 47B35; Secondary 30A78, 46E40, 60G25.

Key words and phrases. Factorization problem, operator valued meromorphic function, operator valued entire function, outer function, shift operator, Toeplitz operator.
} 
1. Shift analysis and factorization. By a shift operator we mean an isometry $S$ on a Hilbert space such that $S^{* n} \rightarrow 0$ strongly as $n \rightarrow \infty$. In this section we fix a Hilbert space $\mathfrak{H C}$ and a shift operator $S$ on $\mathcal{H}$. We write $\mathfrak{e}=\operatorname{ker} S^{*}$. The dimension of $\mathcal{C}$ is called the multiplicity of $S$. The projection mapping $\mathfrak{F}$ onto $\mathfrak{C}$ is denoted $P_{0}$. For any $j=0,1,2, \cdots$, the projection mapping $\mathcal{H C}$ onto $S^{j} \mathfrak{C}$ is given by $P_{j}=S^{j}\left(I-S S^{*}\right) S^{* j}$. Since the projections $P_{0}, P_{1}, P_{2}, \cdots$ are pairwise orthogonal and sum strongly to the identity operator on $\mathfrak{H C}$, we have

$$
\mathfrak{H}=\sum_{0}^{\infty} \oplus S^{j} \mathcal{C}
$$

It is easy to exhibit the reducing subspaces of $S$ in terms of this decomposition. If $\mathfrak{H C}_{0}$ is a subspace of $\mathcal{H C}$ which reduces $S$, then $S_{0}=S \mid \mathcal{H C}_{0}$ is a shift operator on $\mathfrak{H}_{0}$. Hence

$$
\mathfrak{H}_{0}=\sum_{0}^{\infty} \oplus S^{j} \mathcal{C}_{0}
$$

for some subspace $\mathfrak{C}_{0}$ of $\mathcal{C}$. Conversely, any subspace $\mathfrak{H}_{0}$ of $\mathscr{H}$ of this form reduces $S$.

We write $B(\mathcal{H C}), B(\mathcal{H C}, \mathcal{C})$, for example, for the spaces of bounded operators mapping $\mathfrak{H C}$ into $\mathfrak{H}$, respectively $\mathfrak{H C}$ into $\mathfrak{C}$.

We associate with each $T \in B(\mathcal{H C})$ a semi-infinite matrix, $T \sim\left[A_{j k}\right]$, with entries $A_{j k} \in \mathbb{B}(\mathcal{C}), j, k=0,1,2, \cdots$. The action of $T$ is determined by the matrix according to this rule: $T f=g$ where $f=\sum_{0}^{\infty} S^{j} a_{j}$, $g=\sum_{0}^{\infty} S^{j} b_{j}$ and

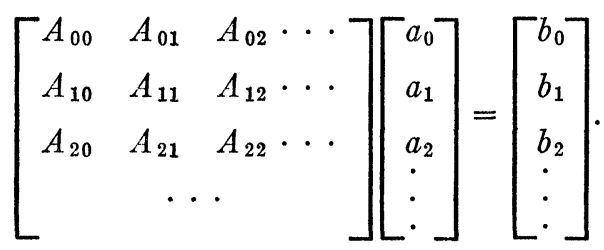

Explicitly,

$$
A_{j k}=P_{0} S^{* j} T S^{k} P_{0} \mid \mathcal{e}, \quad j, k=0,1,2, \cdots .
$$

If $T_{1} \sim\left[A_{j k}\right], \quad T_{2} \sim\left[B_{j k}\right], \quad$ then $T_{1} T_{2} \sim\left[C_{j k}\right]$ where, for each $j, k=0,1,2, \cdots, C_{j k}=\sum_{p=0}^{\infty} A_{j p} B_{p k}$ with convergence in the strong operator topology. This follows from (1) and the fact, already noted, that the projections $P_{p}=S^{p} P_{0} S^{* p}, p=0,1,2, \cdots$, sum strongly to the identity operator on $\mathfrak{F}$.

1.1. Definition. Let $T \in \mathbb{B}(\mathcal{J C})$. We say that $T$ is 
(i) S-Toeplitz if $S^{*} T S=T$,

(ii) $S$-analytic if $T S=S T$,

(iii) $S$-inner if $T$ is $S$-analytic and partially isometric,

(iv) $S$-outer if $T$ is $S$-analytic and CL(T3C) reduces $S$.

It is easy to see that $T$ is $S$-Toeplitz or $S$-analytic if and only if its matrix has the form

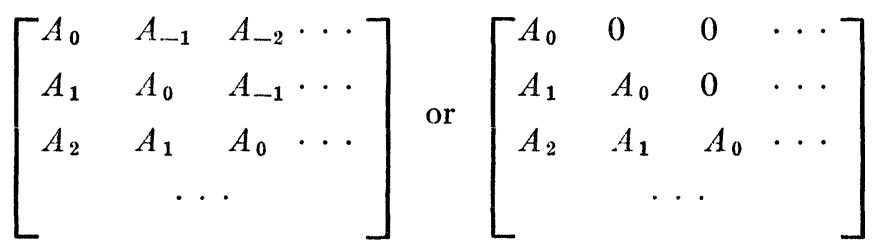

respectively. Then

$$
A_{j}=P_{0} S^{*_{j}} T P_{0} \mid \mathrm{e}, \quad j=0,1,2, \cdots,
$$

and

$$
A_{-j}=P_{0} T S^{j} P_{0} \mid \mathfrak{e}, \quad j=1,2,3, \cdots,
$$

by (1).

We will usually denote $S$-analytic operators by letters $A, B, \cdots$. Then without further explanation we will write $\left(A_{j}\right)_{0}^{\infty},\left(B_{j}\right)_{0}^{\infty}, \cdots$ for the corresponding operators in $B(\mathcal{C})$ which appear in the matrix representations as above.

Notice that the initial space of any $S$-inner operator $B$ reduces $S$. In fact $\operatorname{ker} B$ is invariant under $S$ since $B S=S B$, while for any $f \in H$, $\|B f\|=\|f\|$ implies $\|B S f\|=\|S B f\|=\|B f\|=\|f\|=\|S f\|$. On the other hand, the final space of an $S$-inner operator $B$ reduces $S$ if and only if $B^{*}$ is also $S$-inner. In this degenerate case, $B_{0}$ is a partially isometric operator on $\mathcal{C}$, and $B$ is completely determined by $B_{0}$ according to the formula

$$
B\left(\sum_{0}^{\infty} S^{j} c_{j}\right)=\sum_{0}^{\infty} S^{j}\left(B_{0} c_{j}\right),
$$

where $\left(c_{j}\right)_{0}^{\infty}$ is any sequence in $\mathcal{C}$ such that $\sum_{0}^{\infty}\left|c_{j}\right|^{2}<\infty$.

If $A$ is an $S$-analytic operator on $\mathfrak{H C}$, then $T=A^{*} A$ is $S$-Toeplitz and nonnegative. We are mainly concerned with the problem of finding conditions on a nonnegative $S$-Toeplitz operator such that it will be of this form.

It is convenient to begin with a characterization of products $A A^{*}$ where $A$ is $S$-analytic. This characterization is implicit in de Branges and Rovnyak [4]. See also Sz.-Nagy and Foias [21]. 
1.2. TheOREM. Let $T$ be a nonnegative operator on $\mathfrak{H C}$. Then $T=A A^{*}$ for some $S$-analytic operator $A$ if and only if $T-S T S^{*}=J^{*} J$ for some $J \in B(\mathcal{H C}, \mathcal{C})$. In this case, $A$ is determined by $T$ up to a trivial right factor. More precisely, if $A$ and $C$ are two $S$-analytic operators such that $T=A A^{*}$ and $T=C C^{*}$, then $A=C V, C=A V^{*}$ for some operator $V$ such that both $V$ and $V^{*}$ are $S$-inner, with initial spaces $\sum_{0}^{\infty} \oplus S^{j} \mathcal{C}_{A}$ and $\sum_{0}^{\infty} \oplus S^{j} \mathfrak{C}_{C}$ respectively, where $\mathfrak{e}_{A}=\mathrm{CL}\left(P_{0} A^{*} \mathfrak{H}\right)$ and $\mathfrak{e}_{C}$ $=\mathrm{CL}\left(P_{0} C^{*} \mathfrak{H}\right)$.

Proof. If $T=A A^{*}$ where $A$ is $S$-analytic, then $T-S T S^{*}$ $=A\left(I-S S^{*}\right) A^{*}=A P_{0} A^{*}=J^{*} J$ where $J=P_{0} A^{*} \in \mathbb{B}(\mathcal{H C}, \mathcal{C})$.

Suppose $T-S T S^{*}=J^{*} J$ for some $J \in ß(\mathcal{H C}, \mathcal{C})$. On iterating this identity we obtain

$$
T-S^{n+1} T S^{*+1+1}=\sum_{0}^{n} S^{j} J^{*} J S^{* j}
$$

for all $n=0,1,2, \cdots$. Therefore, if we regard $J$ as an operator from $\mathfrak{H}$ to $\mathfrak{H C}$, we have

$$
\begin{aligned}
\langle T f, g\rangle-\left\langle S^{n+1} T S^{* n+1} f, g\right\rangle & =\sum_{0}^{n}\left\langle J S^{* j} f, J S^{* j} g\right\rangle \\
& =\left\langle\sum_{0}^{n} S^{j} J S^{* j} f, \sum_{0}^{n} S^{k} J S^{* k} g\right\rangle
\end{aligned}
$$

for all $f, g \in \mathcal{H C}, n=0,1,2, \cdots$. Define $A \in ß(\mathcal{H C})$ by $A^{*}=\sum_{0}^{\infty} S^{j} J S^{* j}$. It is easy to see that the series converges in the strong operator topology, $A$ is $S$-analytic, and by the last identity, $\langle T f, g\rangle=\left\langle A A^{*} f, g\right\rangle$ for all $f, g \in \mathcal{H}$. Therefore the condition in the theorem is both necessary and sufficient.

Now let $A$ and $C$ be two $S$-analytic operators such that $A A^{*}=C C^{*}$. If $T$ is the common value of $A A^{*}$ and $C C^{*}$ then

$$
A P_{0} A^{*}=T-S T S^{*}=C P_{0} C^{*}
$$

as above. In particular there is a unique partial isometry $V_{0} \in \mathcal{Q}(\mathcal{C})$ with initial space $\mathcal{C}_{A}=\mathrm{CL}\left(P_{0} A^{*} \mathfrak{J C}\right)$ and final space $\mathfrak{C}_{C}=\mathrm{CL}\left(P_{0} C^{*} \mathfrak{J C}\right)$ such that

$$
V_{0}: P_{0} A^{*} f \rightarrow P_{0} C^{*} f, \quad f \in \mathfrak{H} .
$$

The operator $V_{0}$ has a unique extension to an $S$-analytic operator $V$ on $\mathfrak{H}$, determined by

$$
V S^{j} c=S^{j} V_{0} c, \quad c \in \mathcal{e}, j=0,1,2, \cdots
$$


Clearly $V$ and $V^{*}$ are $S$-inner with initial spaces $\sum_{0}^{\infty} \oplus S^{j} \mathcal{C}_{A}$ and $\sum_{0}^{\infty} \oplus S^{j} \mathcal{C}_{c}$ respectively. By construction $A c=C V c$ whenever $c=P_{0} A^{*} f$ for some $f \in \mathcal{F}$. We find at once that $A g=C V g$ for every $g \in \sum_{0}^{\infty} \oplus S^{j} \mathfrak{C}_{\boldsymbol{A}}$. If $g \perp \sum_{0}^{\infty} \oplus S^{j} \mathfrak{C}_{\boldsymbol{\Lambda}}$ and $g=\sum_{0}^{\infty} S^{j} b_{j}, b_{0}, b_{1}, b_{2}, \cdots$ $\in \mathbb{e}$, then

$$
0=\left\langle g, S^{j} P_{0} A^{*} f\right\rangle=\left\langle A b_{j}, f\right\rangle
$$

for all $f \in \mathcal{F}$. So $A g=\sum_{0}^{\infty} S^{j} A b_{j}=0=C V g$. Hence $A=C V$. Applying a similar argument with the roles of $A$ and $C$ interchanged, we get also $C=A V^{*}$. This completes the proof.

As an application we derive the standard characterization of the invariant subspaces of $S$ in terms of $S$-inner operators.

1.3. TheOREM. $A$ subspace $\mathfrak{T C}$ of $\mathcal{H}$ is invariant under $S$ if and only if $\mathfrak{N}=B \mathfrak{B C}$ for some $S$-inner operator $B$. In this case $B$ is determined by IT up to a trivial right factor as in Theorem 1.2.

1.4. Lemma. If $Q$ is a projection operator acting on a separable Hilbert space $\Re$, then

$$
\operatorname{dim} Q \nVdash=\sum_{j \in J}\left\|Q_{j}\right\|^{2}
$$

for any orthonormal basis $\left\{\phi_{j}\right\}_{j \in J}$ for $\nVdash$.

PROOF OF 1.4. Let $\left\{\psi_{k}\right\}_{k \in K}$ be any orthonormal basis for QK. Then

$$
\begin{aligned}
\sum_{j \in J}\left\|Q \phi_{j}\right\|^{2} & =\sum_{j \in J} \sum_{k \in K}\left|\left\langle Q \phi_{j}, \psi_{k}\right\rangle\right|^{2} \\
& =\sum_{k \in K} \sum_{j \in J}\left|\left\langle\phi_{j}, \psi_{k}\right\rangle\right|^{2} \\
& =\sum_{k \in K}\left\|\psi_{k}\right\|^{2}=\operatorname{dim} Q \mathcal{K} .
\end{aligned}
$$

Proof of 1.3. The range of an $S$-inner operator is clearly a closed invariant subspace for $S$.

Suppose $\mathscr{T}$ is a subspace of $\mathfrak{T C}$ which is invariant under $S$, and let $P$ be the projection mapping $\mathcal{K}$ onto $\mathscr{T}$. We show that $P$ satisfies the condition in Theorem 1.2. Since $P$ is selfadjoint, idempotent, and $P S P=S P$, the operator $Q=P-S P S^{*}$ is selfadjoint and idempotent. Hence $Q$ is also a projection operator. We claim that $\operatorname{dim} Q \mathcal{F} \leqq \operatorname{dim} \mathrm{e}$. If $\mathfrak{C}$ is infinite dimensional, then $\mathfrak{H C}$ has the same dimension as $\mathbb{C}$ and the inequality is trivial. If $\mathcal{C}$ is finite dimensional, then $\mathfrak{H}$ is separable. Let $\left\{e_{k}\right\}_{k \in K}$ be any orthonormal basis for $\mathcal{C}$. Then the vectors $S^{j} e_{k}, k \in K, j=0,1,2, \cdots$, form an orthonormal basis for $\mathcal{H}$. By Lemma 1.4, 


$$
\begin{aligned}
\operatorname{dim} Q \mathcal{H C} & =\sum_{k \in K} \sum_{j=0}^{\infty}\left\langle Q S^{j} e_{k}, S^{j} e_{k}\right\rangle \\
& =\sum_{k \in K} \sum_{j=0}^{\infty}\left\langle\left(P-S P S^{*}\right) S^{j} e_{k}, S^{j} e_{k}\right\rangle \\
& =\lim _{n \rightarrow \infty} \sum_{k \in K}\left\langle P S^{n} e_{k}, S^{n} e_{k}\right\rangle \\
& \leqq \sum_{k \in K}\left\|e_{k}\right\|^{2}=\operatorname{dim} \mathbb{C} .
\end{aligned}
$$

Hence $\operatorname{dim} Q \mathfrak{H} \leqq \operatorname{dim} \mathcal{C}$ in any case.

It follows that there exists an isometry $V$ mapping $Q \mathfrak{H C}$ into $\mathcal{C}$. But then $P-S P S^{*}=Q=J^{*} J$ where $J=V Q \in B(\mathcal{H C}, \mathfrak{e})$. By Theorem $1.2, P=B B^{*}$ for some $S$-analytic operator $B$. Since $P$ is a projection operator, $B$ is $S$-inner. By construction $\mathfrak{N}=P \mathcal{H C}=B \mathcal{H}$.

The uniqueness assertion follows from the corresponding part of Theorem 1.2.

We proceed to a characterization of products $A^{*} A$ where $A$ is an $S$-analytic operator on $\mathfrak{H C}$.

1.5. Definition. A nonnegative $S$-Toeplitz operator $T$ on $\mathfrak{H C}$ is called factorable if $T=A^{*} A$ for some $S$-analytic operator $A$ on $\mathfrak{H C}$.

1.6. Definition. Let $T$ be any nonnegative $S$-Toeplitz operator on $\mathcal{H}$. We write $\mathcal{H}_{T}=\mathrm{CL}\left(T^{1 / 2} \mathcal{H C}\right)$ for the closure of the range of $T^{1 / 2}$, considered as a Hilbert space in the metric of $\mathcal{H}$. We write $S_{T}$ for the unique isometry on $\mathfrak{H}_{T}$ such that $S_{T}\left(T^{1 / 2} f\right)=T^{1 / 2} S f$ for each $f \in \mathfrak{H}$. We put $\mathfrak{C}_{T}=\operatorname{ker} S_{T}^{*}$.

The definition of $S_{T}$ is meaningful because for each $f$ in $\mathfrak{H}$, $\left\|T^{1 / 2} S f\right\|^{2}=\left\langle S^{*} T S f, f\right\rangle=\langle T f, f\rangle=\left\|T^{1 / 2} f\right\|^{2}$.

1.7. Theorem. Let $T$ be a nonnegative $S$-Toeplitz operator on $\mathfrak{H}$. The following are equivalent:

(i) $T$ is factorable,

(ii) $S_{T}$ is a shift operator on $\mathfrak{H}_{T}$,

(iii) there is a dense subspace $\mathfrak{C}_{0}$ of $\mathfrak{e}$ such that

$$
\lim _{n \rightarrow \infty}\left\{\sup \left[\left|\left\langle T c, S^{n} f\right\rangle\right|: f \in \mathscr{H},\langle T f, f\rangle=1\right]\right\}=0
$$

for each $c \in \mathfrak{C}_{0}$. In this case we can write $T=A^{*} A$ where $A$ is $S$-outer and $A_{0} \geqq 0$.

The theorem is a version of Lowdenslager's theorem [17] as presented by Rosenblum [25]. Technically it is different because $\mathfrak{H C}_{\boldsymbol{T}}$ is now defined without a change from the norm of $\mathfrak{H C}$. 
Proof. (i) $\Rightarrow$ (iii). Let $T=A^{*} A$ where $A$ is $S$-analytic. If $c \in \mathfrak{e}$, $f \in \mathcal{F}$, and $\langle T f, f\rangle=1$, then

$$
\begin{aligned}
\left|\left\langle T c, S^{n} f\right\rangle\right|^{2} & =\left|\left\langle A^{*} A c, S^{n} f\right\rangle\right|^{2}=\left|\left\langle S^{*_{n}} A c, A f\right\rangle\right|^{2} \\
& \leqq\left\|S^{*_{n}} A c\right\|^{2}\|A f\|^{2}=\left\|S^{*_{n}} A c\right\|^{2}\langle T f, f\rangle=\left\|S^{*_{n}} A c\right\|^{2} .
\end{aligned}
$$

It follows that (iii) holds with $\mathfrak{C}_{0}=\mathfrak{e}$.

(iii) $\Rightarrow$ (ii). Suppose (iii) holds. For each $c \in \mathcal{C}_{0}$ and $n=0,1,2, \cdots$,

$$
\left\|S_{T}^{* n} T^{1 / 2} c\right\|=\sup \left[\left|\left\langle T c, S^{n} f\right\rangle\right|: f \in \mathcal{F},\langle T f, f\rangle=1\right] .
$$

This follows from the fact that $\left\langle T c, S^{n} f\right\rangle=\left\langle T^{1 / 2} c, T^{1 / 2} S^{n} f\right\rangle=\left\langle T^{1 / 2} c\right.$, $\left.S_{T}^{n} T^{1 / 2} f\right\rangle=\left\langle S_{T}^{* n} T^{1 / 2} c, T^{1 / 2} f\right\rangle$, and, the set of vectors $T^{1 / 2} f, f \in \mathcal{H}$, $\langle T f, f\rangle=1$, is dense in the unit sphere in $\mathcal{F}_{T}$. Therefore if (iii) holds, then

$$
\lim _{n \rightarrow \infty}\left\|S_{T}^{* n} g\right\|=0
$$

for any $g$ in $\mathfrak{F C}_{T}$ of the form $g=T^{1 / 2} c, c \in \mathcal{C}_{0}$. If $g=T^{1 / 2} S^{k} c$, for some $c \in \mathcal{C}_{0}$ and $k=0,1,2, \cdots$, then $S_{T}^{* n} g=S_{T}^{* n} T^{1 / 2} S^{k} c=S_{T}^{* n} S_{T}^{k} T^{1 / 2} c$ $=S_{T}^{* n-k} T^{1 / 2} c$ if only $n \geqq k$; hence (2) holds for such $g$. By linearity and approximation, (2) holds for all $g$ in $\mathcal{F}_{T}$. Hence $S_{T}$ is a shift operator and (ii) holds.

(ii) $\Rightarrow$ (i). Assume that $S_{T}$ is a shift operator. Define $J \in B\left(\mathcal{C}_{T}, \mathfrak{e}\right)$ by $J k=T^{1 / 2} k, k \in \mathcal{C}_{T}$. To see that $T^{1 / 2} \mathcal{C}_{T} \subseteq \mathcal{C}$, note that by the definition of $S_{T}, T^{1 / 2} S_{T}^{*} g=S^{*} T^{1 / 2} g, g \in \mathcal{H C}_{T}$; hence if $k \in \mathcal{C}_{T}=\operatorname{ker} S_{T}^{*}$ then $T^{1 / 2} k \in \mathcal{C}=\operatorname{ker} S^{*}$. Thus $J$ is well defined. Clearly $J$ is one-to-one. Therefore in the polar decomposition $J=R V, R=\left(J J^{*}\right)^{1 / 2}, V$ is an isometry mapping $\mathcal{C}_{T}$ into $\mathcal{C}$. Since we assume that $S_{T}$ is a shift operator, each $g \in \mathcal{F C}_{T}$ has a unique representation

$$
g=\sum_{0}^{\infty} S_{T}^{j} k_{j}
$$

where $k_{j} \in \mathcal{C}_{T}, j=0,1,2, \cdots$. Therefore we can define an isometry $K$ mapping $\mathcal{H}_{T}$ into $\mathcal{H}$ by setting

$$
K g=\sum_{0}^{\infty} S^{j}\left(V k_{j}\right)
$$

We have then $K S_{T}=S K$. Now define $A \in B(\mathcal{H C})$ by $A f=K T^{1 / 2} f, f \in \mathcal{H}$. For any $f \in \mathcal{F}, A S f=K T^{1 / 2} S f=K S_{T} T^{1 / 2} f=S K T^{1 / 2} f=S A f$, and $\langle T f, f\rangle$ $=\left\langle T^{1 / 2} f, T^{1 / 2} f\right\rangle=\left\langle K T^{1 / 2} f, K T^{1 / 2} f\right\rangle=\langle A f, A f\rangle$. Thus $A$ is $S$-analytic and $T=A^{*} A$, so (i) holds.

It is easily checked that for the operator $A$ just constructed 
$\mathrm{CL}(A \mathfrak{F C})=\mathrm{CL}\left(K T^{1 / 2} \mathfrak{F C}\right)=K \mathfrak{F C}_{T}$ reduces $S$ so $A$ is $S$-outer. Also $A_{0}=P_{0} A P_{0} \mid \mathrm{e}=R \geqq 0$. This completes the proof.

The condition $A_{0} \geqq 0$ was used by Masani [18] to discuss uniqueness of factors.

1.8. TheOREM. Let $T$ be a nonnegative $S$-Toeplitz operator. If $T$ is factorable, then there is a unique $S$-outer operator $A$ such that $T=A^{*} A$ and $A_{0} \geqq 0$.

We make use of a second uniqueness result.

1.9. Theorem. Let $T$ be a nonnegative $S$-Toeplitz operator. Suppose $T$ is factorable and let $T=A^{*} A$ and $T=C^{*} C$ be two representations where $A$ and $C$ are $S$-outer operators. Then $A$ and $C$ are equivalent in the sense that $A=V C, C=V^{*} A$ where $V$ is an operator such that both $V$ and $V^{*}$ are S-inner with initial spaces $\mathrm{CL}(C \mathrm{C})$ and $\mathrm{CL}(A \mathrm{H})$ respectively. Conversely if $A$ and $C$ are equivalent $S$-outer operators then $A^{*} A=C^{*} C$.

1.10. Lemma. If $A$ is an S-outer operator, then $\mathrm{CL}(A \mathfrak{F})$ $=\sum_{0}^{\infty} \oplus S^{j} \mathrm{CL}\left(A_{0} \mathfrak{e}\right)$.

Proof of 1.10. If $f=A g \in A \mathcal{F}$, we can write $f=A c+A S S^{*} g$ for some $c \in \mathcal{C}$. Then $P_{0} f=P_{0} A c \in A_{0} \mathrm{e}$ because $A_{0}=P_{0} A P_{0} \mid \mathcal{C}$ by (1). Therefore $P_{0} A \mathfrak{F} \subseteq A_{0} \mathrm{C}$ and $P_{0} \mathrm{CL}(A \mathfrak{F}) \subseteq \mathrm{CL}\left(A_{0} \mathcal{e}\right) \subseteq P_{0} \mathrm{CL}(A \mathfrak{F})$, hence $P_{0} \mathrm{CL}(A \mathfrak{F})=\mathrm{CL}\left(A_{0} \mathcal{C}\right)$. Since $A$ is $S$-outer, $\mathrm{CL}(A \mathfrak{F})$ reduces $S$ so

$$
\mathrm{CL}(A \mathfrak{F})=\sum_{0}^{\infty} \oplus S^{j} \mathbb{C}_{0}
$$

where $\mathfrak{C}_{0}=\operatorname{ker}\left(S^{*} \mid \mathrm{CL}(A \mathfrak{F})\right)=P_{0} \mathrm{CL}(A \mathfrak{F})=\mathrm{CL}\left(A_{0} \mathfrak{C}\right)$.

Proofs of 1.8 AND 1.9. Let $A$ and $C$ be $S$-outer operators such that $A^{*} A=C^{*} C$. Define $V$ on $C \mathcal{H C}$ to $\mathcal{H C}$ by $V C f=A f, f \in \mathcal{F}$. Since $A^{*} A$ $=C^{*} C, V$ is well defined and has a unique extension to a partial isometry on $\mathfrak{F}$, also denoted $V$, with initial space $\mathrm{CL}(C \mathfrak{C})$ and final space CL $(A \mathcal{F})$. We find at once that both $V$ and $V^{*}$ commute with $S$, so both are $S$-inner. The first assertion in Theorem 1.9 follows; the second is verified by direct calculation.

Suppose now that $A_{0} \geqq 0, C_{0} \geqq 0$. Since $A=V C, C=V^{*} A$, we have $A_{0}=V_{0} C_{0}, \quad C_{0}=V_{0}^{*} A_{0}$. Hence $A_{0}^{2}=C_{0} V_{0}^{*} V_{0} C_{0} \leqq C_{0}^{2}$ and $C_{0}^{2}$ $=A_{0} V_{0} V_{0}^{*} A_{0} \leqq A_{0}^{2}$, so $A_{0}^{2}=C_{0}^{2}$. Since $A_{0} \geqq 0, C_{0} \geqq 0$, we have $A_{0}=C_{0}$. Since $A_{0}=V_{0} C_{0}, V_{0}$ is the identity on $\mathrm{CL}\left(C_{0} \mathcal{e}\right)$. Hence $V$ is the identity on $\mathrm{CL}\left(C_{0} \mathrm{e}\right)$. Since $V$ commutes with $S$ and $\mathrm{CL}\left(C_{\mathfrak{F}}\right)$ $=\sum_{0}^{\infty} \oplus S^{j} \mathrm{CL}\left(C_{0} \mathcal{e}\right)$ by Lemma 1.10 , it follows that $V$ is the identity on $\mathrm{CL}(C H C)$. But $A=V C$, so we get $A=C$. This proves the unique- 
ness part of Theorem 1.8. The existence assertion follows from Theorem 1.7.

1.11. Theorem. Let $A$ be an S-analytic operator. Then $A$ has a unique factorization $A=B C$ where $C$ is $S$-outer, $C_{0} \geqq 0$, and $B$ is $S$-inner with initial space $\mathrm{CL}(C H C)$. For this choice of $C, C^{*} C=A^{*} A$. On the other hand, if $C$ is any $S$-outer operator such that $C^{*} C=A^{*} A$, then $A=B C$ for some $S$-inner operator $B$ with initial space $\mathrm{CL}(C H C)$.

Proof. Consider any $S$-outer operator $C$ such that $C^{*} C=A^{*} A$. By Theorem 1.8 there is a unique such $C$ with $C_{0} \geqq 0$. But for any such $C$ there is a unique partial isometry $B$ on $\mathcal{H}$ with initial space $\mathrm{CL}(C \mathcal{H C})$ such that $B(C f)=A f, f \in \mathcal{H}$, i.e. $A=B C$. If $g=C f \in C \mathcal{H}$ then $B S g$ $=B C S f=A S f=S A f=S B g$. Since $C$ is $S$-outer, $C L(C F)$ reduces $S$. Therefore if $g \perp C \mathfrak{F}$ then $S g \perp C \mathcal{F}$ and $B S g=0=S B g$. It follows that $B$ is $S$-inner. The assertions of the theorem follow quickly from these facts.

The $S$-outer operators are distinguished from more general $S$-analytic operators by their extremal properties. Our first result in this direction is motivated by work of Lax [15] and Masani [18].

1.12. TheOREM. Each of the following conditions is necessary and sufficient for an $S$-analytic operator $C$ to be $S$-outer:

(i) $\mathrm{CL}(C H \mathrm{C})=\sum_{0}^{\infty} \oplus S^{j} \mathrm{CL}\left(C_{0} \mathrm{e}\right)$,

(ii) for each $c \in \mathfrak{C},\left\langle C_{0}^{*} C_{0} c, c\right\rangle=\inf _{f \in 3 c}\left\langle C^{*} C(c-S f), c-S f\right\rangle$,

(iii) $C_{0}{ }^{*} C_{0} \geqq A_{0}^{*} A_{0}$ for every $S$-analytic operator $A$ such that $C^{*} C$ $=A^{*} A$.

Proof. Let (o) be the condition that $C$ is $S$-outer.

$(o) \Leftrightarrow(i)$. Sufficiency is obvious; necessity follows from Lemma 1.10.

(o) $\Rightarrow$ (ii). If $C$ is $S$-outer and $c \in \mathcal{e}$, then $S^{*} C c \in C L(C J C)$ and the vector $g=S^{*} C c$ minimizes $\|C c-S g\|$. Hence

$$
\begin{aligned}
& \inf _{f \in \Im C}\left\langle C^{*} C(c-S f), c-S f\right\rangle=\inf _{f \in \Im C}\|C c-S C f\|^{2} \\
& =\inf _{0 \in \mathrm{CL}(C \Im))}\|C c-S g\|^{2}=\left\|P_{0} C c\right\|^{2} \\
& =\left\langle C_{0}^{*} C_{0} c, c\right\rangle \text {. }
\end{aligned}
$$

(ii) $\Rightarrow$ (iii). If (ii) holds and $A$ is $S$-analytic with $C^{*} C=A^{*} A$, then, for any $c \in \mathfrak{E}$,

$$
\begin{aligned}
& \left\langle C_{0}^{*} C_{0} c, c\right\rangle=\inf _{\mathcal{f} \in \mathfrak{H C}}\left\langle A^{*} A(c-S f), c-S f\right\rangle=\inf _{\mathcal{r} \in \mathfrak{K C}}\|A c-S A f\|^{2} \\
& \geqq \inf _{0 \in \mathscr{C} C}\|A c-S g\|^{2}=\left\|P_{0} A c\right\|^{2}=\left\langle A_{0}^{*} A_{0} c, c\right\rangle \text {. }
\end{aligned}
$$


(iii) $\Rightarrow$ (i). Assume (iii) holds. Factor $C=B A$ where $A$ is $S$-outer and $B$ is $S$-inner with initial space $\mathrm{CL}(A \mathcal{F C})$. This is possible by Theorem 1.11. Then $C^{*} C=A^{*} A$, so by (iii), $C_{0}^{*} C_{0} \geqq A_{0}^{*} A_{0}$. But $C_{0}=B_{0} A_{0}$ where $\left\|B_{0}\right\| \leqq 1$. Hence $C_{0}^{*} C_{0}=A_{0}^{*} A_{0}$. It follows that $B_{0}$ is isometric on $A_{0} \mathrm{e}$. Since $B_{0}=P_{0} B P_{0} \mid \mathcal{C}, B$ agrees with $B_{0}$ on $A_{0} \mathfrak{e}$, and therefore $B S^{j}$ agrees with $S^{j} B_{0}$ on $\mathrm{CL}\left(A_{0} \mathcal{C}\right), j=0,1,2, \cdots$. Hence

$$
\begin{gathered}
B S^{j} \mathrm{CL}\left(A_{0} \mathfrak{e}\right)=S^{j} B_{0} \mathrm{CL}\left(A_{0} \mathfrak{e}\right)=S^{j} \mathrm{CL}\left(C_{0} \mathfrak{e}\right) \\
\mathrm{CL}(C \mathfrak{C})=B \mathrm{CL}(A \mathfrak{F})=B\left(\sum_{0}^{\infty} \oplus S^{j} \mathrm{CL}\left(A_{0} \mathfrak{e}\right)\right)=\sum_{0}^{\infty} \oplus S^{j} \mathrm{CL}\left(C_{0} \mathfrak{e}\right) .
\end{gathered}
$$

Therefore (iii) implies (i), and each of the conditions (i)-(iii) is equivalent to $C$ being $S$-outer.

Some interesting variants of condition (iii) in Theorem 1.12 are suggested by a result of Robinson [23] (see Theorem 2.6). We now extend Robinson's ideas.

1.13. TheOREM. Let $C$ be an S-analytic operator. Let w be any subset of the double commutant of $S$ such that the weakly closed linear span of $W$ and the identity operator contains $S$. Let $\mathfrak{F}$ be any subset of $3 \mathrm{C}$ such that $P_{0} \mathcal{F}$ spans a dense subspace of $\mathcal{C}$. Then $C$ is $S$-outer if and only if

$$
\left\|W^{*} C f\right\| \leqq\left\|W^{*} A f\right\|
$$

for all $W \in W, f \in F$, and every $S$-analytic operator $A$ such that $A^{*} A$ $=C^{*} C$.

In particular if $C$ is $S$-outer, then (3) holds for every operator $W$ in the double commutant of $S$, all $f \in \mathcal{H}$, and every $S$-analy tic operator $A$ such that $A^{*} A=C^{*} C$. The proof shows more, and for reference purposes we isolate some additional facts in

1.14. Lemma. Let $B$ be an S-inner operator with initial space $\Re$.

(i) If $g \in \mathfrak{N}$ and $W$ is any $S$-analytic operator which commutes with $B$, then

$$
\left\|W^{*} g\right\| \leqq\left\|W^{*} B g\right\|
$$

with equality if and only if $B W^{*} g=W^{*} B g$.

(ii) Let $W$ be any set of $S$-analytic operators which commute with $B$ such that the weakly closed linear span of w and the identity operator contains $S$. Let $\mathcal{G}$ be any subset of $\mathfrak{T}$ such that $P_{0} \mathcal{G}$ spans a dense subspace of $P_{0} \mathrm{TT}$. If equality holds in (4) for all $W \in W$ and all $g \in \mathcal{G}$, then $B^{*}$ is also $S$-inner.

In Theorem 1.13 or Lemma 1.14 we could choose $W=\left\{V^{t}\right\}_{t>0}$, where $V^{t}=\exp \left[-t(I+S)(I-S)^{-1}\right]$ for each $t>0$. The exponential is 
defined to be the strong limit of $\exp \left[-t(I+r S)(I-r S)^{-1}\right]$ as $r \nearrow 1$. See Sz.-Nagy and Foiaş [21]. A short calculation gives

$$
r S=I-2 \int_{0}^{\infty} e^{-t} \exp \left[-t(I+r S)(I-r S)^{-1}\right] d t
$$

for $0<r<1$, where the integral is taken in the weak sense. It follows that

$$
S=I-2 \int_{0}^{\infty} e^{-t} V^{t} d t .
$$

The last integral can also be taken as an improper Riemann in tegral with convergence in the uniform operator topology. In particular, the weakly closed linear span of $W=\left\{V^{t}\right\}_{t>0}$ and $I$ contains $S$. See Theorem 2.6.

A different choice of $w$ leads to

1.15. Theorem. Let $C$ be an $S$-analytic operator. Then $C$ is $S$-outer if and only if

$$
\sum_{0}^{n} C_{j}^{*} C_{j} \geqq \sum_{0}^{n} A_{j}^{*} A_{j}, \quad n=0,1,2, \cdots,
$$

for every $S$-analytic operator $A$ such that $A^{*} A=C^{*} C$. More generally, (5) holds if $A$ and $C$ are any $S$-analytic operators which are related by $A=B C$ for some $S$-inner operator $B$ whose initial space contains $\mathrm{CL}(C \mathfrak{H})$.

ProOF of 1.14. Let $g \in \mathfrak{M}$ and let $W$ be any $S$-analytic operator which commutes with $B$. Then $B^{*} B g=g$ and so

$$
\left\|W^{*} g\right\|=\left\|W^{*} B^{*} B g\right\|=\left\|B^{*} W^{*} B g\right\| \leqq\left\|W^{*} B g\right\| \text {. }
$$

Since $B^{*}$ is a partial isometry, equality holds only if $W^{*} B g=B h$ for some $h \in \Re$. Then $B^{*} B h=h$, and since $W$ commutes with $B$, we get $W^{*} g=h$. Hence $W^{*} B g=B W^{*} g$. Conversely if $W^{*} B g=B W^{*} g$ then $\left\|W^{*} B g\right\|=\left\|B W^{*} g\right\| \leqq\left\|W^{*} g\right\| \leqq\left\|W^{*} B g\right\|$, and equality holds throughout. This proves (i).

To prove (ii) note that, by (i), $B W^{*} g=W^{*} B g$ for all $W \in W$, $g \in \mathcal{G}$. By our assumptions on $W$ it follows that $B S^{*} g=S^{*} B g, g \in \mathcal{G}$. Multiply this by $S$ and use the fact that $P_{0}=I-S S^{*}$ to get $B P_{0} g$ $=P_{0} B g, g \in \mathcal{G}$. If we set $\mathcal{C}_{0}=P_{0} \mathscr{T}$, so that $\mathscr{T}=\sum_{0}^{\infty} \oplus S^{j} \mathfrak{C}_{0}$, then our assumptions concerning $\mathcal{G}$ imply that $B c=P_{0} B c$ for all $c \in \mathcal{C}_{0}$ and hence for all $c \in \mathfrak{C}$. But then $B$ agrees with $B_{0}=P_{0} B P_{0} \mid \mathfrak{e}$ on $\mathfrak{e}$ and so $B S^{j}$ with $S^{j} B_{0}$ on $S^{j} \mathcal{C}, j=0,1,2, \cdots$. It follows that $B_{0}$ is a par- 
tially isometric operator on $\mathcal{e}$, and $B\left(\sum_{0}^{\infty} S^{j} c_{j}\right)=\sum_{0}^{\infty} S^{j}\left(B_{0} c_{j}\right)$ for any sequence $\left(c_{j}\right)_{0}^{\infty}$ in $e$ such that $\sum_{0}^{\infty}\left|c_{j}\right|^{2}<\infty$. We quickly deduce (ii).

Proof of 1.13. Suppose that $C$ is $S$-outer, and let $A$ be any $S$ analytic operator such that $A^{*} A=C^{*} C$. By Theorem 1.11, $A=B C$ for some $S$-inner operator $B$ with initial space $\mathrm{CL}(C \mathfrak{C})$. If $W$ is any operator in the double commutant of $S$ and $f$ is any vector in $\mathfrak{K}$, we get (3) from (4) on setting $g=C f$.

Conversely suppose that (3) holds for all $W \in$ w, $f \in F$. Factor $C=B A$ where $A$ is $S$-outer and $B$ is $S$-inner with initial space $\mathfrak{T}=\mathrm{CL}(A \mathfrak{F})$. By Theorem 1.12 (i), $P_{0} \mathfrak{T}=\mathrm{CL}\left(A_{0} \mathcal{C}\right)$. Set $\mathcal{G}=P_{0} \mathfrak{F}$. Since $P_{0} A f=A_{0} P_{0} f, f \in \mathfrak{H}$, our assumptions on $\mathcal{F}$ imply that $P_{0} \mathcal{G}$ spans a dense subspace of $P_{0} \mathfrak{T}$. By (3), $\left\|W^{*} B g\right\| \leqq\left\|W^{*} g\right\|$ for all $W \in$ w, $g \in g$. Since the reverse inequality holds automatically by Lemma 1.14 (i), by Lemma 1.14 (ii), $B^{*}$ is also $S$-inner. It follows that $\mathrm{CL}(C \mathfrak{H C})=B \mathrm{CL}(A \mathfrak{H C})$ is the initial space of an $S$-inner operator, hence $\mathrm{CL}(C \mathrm{CH})$ reduces $S$ and $C$ is $S$-outer.

Proof of 1.15. In Theorem 1.13 choose $\rightsquigarrow=\left\{S^{n+1}\right\}_{0}^{\infty}$ and $\mathcal{F}=\mathcal{C}$. Thus $C$ is $S$-outer if and only if

$$
\left\|S^{* n+1} C c\right\| \leqq\left\|S^{* n+1} A c\right\|
$$

for each $n=0,1,2, \cdots, c \in \mathcal{e}$, and every $S$-analytic operator $A$ such that $A^{*} A=C^{*} C$. Thus $C$ is $S$-outer if and only if, for each such $A$ and $n=0,1,2, \cdots$,

$$
P_{0} C^{*} S^{n+1} S^{* n+1} C P_{0} \leqq P_{0} A^{*} S^{n+1} S^{* n+1} A P_{0}
$$

or

$$
P_{0} C^{*}\left(I-\sum_{0}^{n} S^{j} P_{0} S^{* j}\right) C P_{0} \leqq P_{0} A^{*}\left(I-\sum_{0}^{n} S^{j} P_{0} S^{* j}\right) A P_{0} .
$$

This last inequality is equivalent to (5). It is clear from the proof that we can verify (5) for any $S$-analytic operators $A$ and $C$ such that $A=B C$ for some $S$-inner operator $B$ whose initial space contains $\mathrm{CL}(C \mathfrak{H C})$.

We turn again to the problem of giving conditions on a nonnegative $S$-Toeplitz operator $T$ that $T$ be factorable. Theorem 1.7 gives conditions which are both necessary and sufficient. It follows, for example, that if $T$ is $S$-Toeplitz and $T \geqq \delta I$ for some $\delta>0$, then $T$ is factorable. For then $\mathfrak{H C}_{T}=\mathcal{H C}_{C}$ and $S_{T}=T^{1 / 2} S T^{-1 / 2}$; hence $S_{T}^{* n} \rightarrow 0$ strongly and $S_{T}$ is a shift operator. Thus $T$ is factorable. However, the conditions in Theorem 1.7 are not always so easy to apply. It is therefore of interest to have sufficient conditions which are useful for applications. 
The next result is central in our work.

1.16. Theorem. Let $T$ be a nonnegative $S$-Toeplitz operator. Suppose that there exists an operator $V \neq 0$ in the double commutant of $S$ such that $T V$ is $S$-analytic. Then $T$ is factorable. Moreover if $T=A^{*} A$ where $A$ is $S$-outer, then $A^{*} V$ is also $S$-analytic.

1.17. Lemma. Let $V \neq 0$ be an operator in the double commutant of $S$. Then $V$ has a factorization $V=U W$ where $U$ is an isometry in the double commutant of $S$ and $W$ is an $S$-outer operator in the double commutant of $S$.

PROOF OF 1.17. Let $V \sim\left[V_{j-k}\right]$ where $V_{j}=0$ for all $j=-1,-2, \cdots$. Since $V$ is in the double commutant of $S$, there exist scalars $v_{j}$ such that $V_{j}=v_{j} I, j=0, \pm 1, \pm 2, \cdots$. Let $S^{(1)}$ be a shift operator on a Hilbert space $\mathfrak{F}^{(1)}$ such that $\mathfrak{C}^{(1)}=\operatorname{ker} S^{(1) *}$ has dimension 1 . Construct an $S^{(1)}$-analytic operator $V^{(1)}$ such that $V^{(1)} \sim\left[v_{j-k}\right]$. Factor $V^{(1)}=U^{(1)} W^{(1)}$ where $U^{(1)}$ is $S^{(1)}$-inner and $W^{(1)}$ is $S^{(1)}$-outer. Let $U^{(1)} \sim\left[u_{j-k}\right], W^{(1)} \sim\left[w_{j-k}\right]$ where $u_{j}=w_{j}=0$ for $j=-1,-2, \cdots$. Then there exist operators $U, W$ on $\mathscr{H}$ such that $U \sim\left[U_{j-k}\right], W$ $\sim\left[W_{j-k}\right]$ where $U_{j}=u_{j} I, W_{j}=w_{j} I$ for all $j=0, \pm 1, \pm 2, \cdots$, and these operators have the required properties.

Proof of 1.16. Using Lemma 1.17 we can reduce the theorem to the case where $V$ is an isometry.

Suppose then that $V^{*} V=I$. We show that $V^{*} T V=T$. Since $T+I \geqq I$, by a previous remark we can write $T+I=A^{*} A$ where $A$ is $S$-analytic. Since $V^{*} V=I$ and $A V=V A$ by hypothesis, $V^{*} T V$ $=V^{*} A^{*} A V-V^{*} V=A^{*} A-I=T$, as claimed.

Since $T V$ is $S$-analytic, $(T V) S^{n}=S^{n}(T V)$ and $S^{* n} V^{*} T=V^{*} T S^{*_{n}}$ for each $n=0,1,2, \cdots$. Hence if $c \in \mathcal{C}$ and $f \in \mathcal{F},\langle T f, f\rangle=1$, then

$$
\begin{aligned}
\left\langle T c, S^{n} f\right\rangle & =\left\langle S^{*_{n}} T c, f\right\rangle=\left\langle S^{*_{n}} V^{*} T V c, f\right\rangle \\
& =\left\langle V^{*} T S^{*_{n}} V c, f\right\rangle=\left\langle T^{1 / 2} S^{*_{n}} V c, T^{1 / 2} V f\right\rangle,
\end{aligned}
$$

so

$$
\begin{aligned}
\left|\left\langle T c, S^{n} f\right\rangle\right| & \leqq\left\|T^{1 / 2}\right\|\left\|S^{*_{n}} V c\right\|\left\|T^{1 / 2} V f\right\| \\
& =\left\|T^{1 / 2}\right\|\left\|S^{*_{n}} V c\right\|\langle T f, f\rangle \\
& =\left\|T^{1 / 2}\right\|\left\|S^{*_{n}} V c\right\| .
\end{aligned}
$$

Therefore $T$ satisfies condition (iii) of Theorem 1.7, so $T$ is factorable.

Let $T=A^{*} A$ where $A$ is $S$-outer. Since $\left[\left(A^{*} V\right) S-S\left(A^{*} V\right)\right] A$ $=A^{*} A V S-S A^{*} A V=(T V) S-S(T V)=0$, we have $\left(A^{*} V\right) S f$ $=S\left(A^{*} V\right) f$ for all $f \in A \mathfrak{K C}$. But if $f \perp A \mathfrak{K C}$ then $S f \perp A \mathfrak{K}$ and $V S f \perp A \mathfrak{K C}$ 
because $\mathrm{CL}(A \mathcal{F C})$ reduces $S$ and hence also $V$. Therefore $\left(A^{*} V\right) S f=0$ $=S\left(A^{*} V\right) f$ for $f \perp A$ F . Hence $A^{*} V$ is $S$-analytic.

We prove next a comparison theorem which is adapted from B. Moore [19]. Similar results were obtained by Lowdenslager [17] and Douglas [7].

1.18. TheOREM. Let $T_{1}, T_{2}$ be nonnegative $S$-Toeplitz operators such that $T_{1} \leqq T_{2}$. Assume that

$$
\lim _{n \rightarrow \infty}\left\langle T_{2} f_{n}, f_{n}\right\rangle=0
$$

for every sequence $\left(f_{n}\right)$ in $\operatorname{He}$ such that $\lim _{n, k \rightarrow \infty}\left\langle T_{2}\left(f_{n}-f_{k}\right), f_{n}-f_{k}\right\rangle=0$ and $\lim _{n \rightarrow \infty}\left\langle T_{1} f_{n}, f_{n}\right\rangle=0$. Then if $T_{1}$ is factorable so is $T_{2}$.

Proof. Since $\left\|T_{1}^{1 / 2} f\right\|^{2}=\left\langle T_{1} f, f\right\rangle \leqq\left\langle T_{2} f, f\right\rangle=\left\|T_{2}^{1 / 2} f\right\|^{2}, f \in \mathfrak{F C}$, there is a unique operator $C$ mapping $\mathfrak{F C}_{T_{2}}=\mathrm{CL}\left(T_{2}^{1 / 2} \mathfrak{F C}\right)$ into $\mathfrak{H C}_{T_{1}}=\mathrm{CL}\left(T_{1}^{1 / 2} \mathfrak{H C}\right)$ such that

$$
C T_{2}^{1 / 2} f=T_{1}^{1 / 2} f, \quad f \in \mathfrak{T} \text {. }
$$

Our assumptions imply that $C$ has zero kernel and hence $C^{*}$ has range dense in $\mathfrak{K}_{T_{2}}$. For any $f \in \mathfrak{H C}$ and $n=0,1,2, \cdots$,

$$
C S_{T_{2}}^{n} T_{2}^{1 / 2} f=C T_{2}^{1 / 2} S^{n} f=T_{1}^{1 / 2} S^{n} f=S_{T_{1}}^{n} T_{1}^{1 / 2} f=S_{T_{1}}^{n} C T_{2}^{1 / 2} f .
$$

Hence $C S_{T_{2}}^{n}=S_{T_{1}}^{n} C$ and $S_{T_{2}}^{*_{n}} C^{*}=C^{*} S_{T_{1}}^{*_{n}}$ for each $n=0,1,2, \cdots$. If $T_{1}$ is factorable then $S_{T_{1}}$ is a shift operator on $\mathcal{F}_{T_{1}}$ by Theorem 1.7. But then $\left\|S_{T_{2}}^{* n} g\right\| \rightarrow 0$ for each $g$ in the range of $C^{*}$. Since the range of $C^{*}$ is dense in $\mathcal{F C}_{T_{2}}, S_{T_{2}}^{*_{n}} \rightarrow 0$ strongly, so $S_{T_{2}}$ is a shift operator and $T_{2}$ is factorable by Theorem 1.7.

2. Operator valued functions on a circle or line. In the rest of the paper, $\mathcal{e}$ will denote a fixed separable Hilbert space. We write $|\cdot|$ for the norm in both $\mathcal{C}$ and $B(\mathcal{C})$. By a vector or operator valued function we mean a function with values in $\mathcal{C}$ or $B(\mathcal{C})$ respectively. We are mainly concerned with functions defined on the unit circle $\Gamma=\left\{e^{i \theta}: 0 \leqq \theta<2 \pi\right\}$ in the complex plane or the real line $R$. Unless otherwise stated, we use the weak definitions of analyticity and measurability for vector and operator valued functions and integrals of such functions. Measure theoretic notions always refer to the Borel subsets of $\Gamma$ and $R$, normalized Lebesgue measure on $\Gamma$, and Lebesgue measure on $R$. In general we do not distinguish functions which differ only on a null set.

We take for granted (1) basic complex function theory, as presented in Hoffman [12] or Privalov [22], (2) elementary properties of Fou- 
rier series and integrals for vector valued functions, and (3) the definitions and basic properties of the Lebesgue and Hardy classes $L_{\mathfrak{e}}^{2}, H_{\mathfrak{e}}^{2}$ and $L_{\mathscr{B}(e)}^{\infty}, H_{\mathbb{Q}(\mathrm{e})}^{\infty}$ on both $\Gamma$ and $R$. We recall, at least, the definition of $H_{\mathbb{B}(\mathfrak{e})}^{\infty}$ : this is the class of all $F \in L_{\mathbb{Q}(\mathfrak{e})}^{\infty}$ such that $F H_{\mathfrak{e}}^{2} \subseteq H_{\mathrm{e}}^{2}$. We have then $F \in H_{\mathscr{B}(\mathcal{C})}^{\infty}(\Gamma)$ (respectively $F \in H_{\mathbb{B}(e)}^{\infty}(R)$ ) if and only if $F$ is the a.e. strong boundary value function of a bounded operator valued analytic function defined in the disk $|z|<1$ (respectively the halfplane $y>0$ ). There is a similar characterization of $H_{\mathrm{e}}^{2}$ on $\Gamma$ or $R$. Thus, although the elements of $H_{\mathfrak{e}}^{2}$ and $H_{\mathbb{B}(e)}^{\infty}$ are functions defined on $\Gamma$ or $R$, we can and frequently will refer to values of such functions in the disk $|z|<1$ or half-plane $y>0$.

Henceforth $S$ will denote the standard shift operator "multiplication by $e^{i \theta}$ " in $H_{\mathrm{e}}^{2}(\Gamma)$ or "multiplication by $(x-i) /(x+i)$ " in $H_{\mathrm{e}}^{2}(R)$. In $H_{\mathrm{e}}^{2}(\Gamma)$, ker $S^{*}$ is the set of constant functions, and in $H_{\mathrm{e}}^{2}(R)$, ker $S^{*}$ is the set of functions of the form $k(x)=c /(x+i), c \in \mathcal{C}$. In either case $S$ has multiplicity equal to the dimension of $\mathrm{e}$. In fact there is a natural way to identify $\operatorname{ker} S^{*}$ with $\mathcal{C}$ in either case.

An operator $T \in \mathbb{B}\left(H_{\mathfrak{e}}^{2}\right)$ is $S$-Toeplitz if and only if $T f=P F f, f \in H_{\mathfrak{e}}^{2}$, for some $F \in L_{\circledast}^{\infty}(\mathfrak{e})$, where $P$ is the projection mapping $L_{\mathfrak{e}}^{2}$ onto $H_{\mathfrak{e}}^{2}$. In this case $F$ is essentially determined by $T$ and we write $T=T_{F}$. We have $T_{F}^{*}=T_{F^{*}}$, and $T_{F} \geqq 0$ if and only if $F \geqq 0$ a.e. An operator $A \in \mathbb{B}\left(H_{\mathfrak{e}}^{2}\right)$ is $S$-analytic if and only if $A=T_{G}$ for some $G \in H_{\mathfrak{B}(\mathfrak{e})}^{\infty}$, in which case

$$
A f=G f, \quad f \in H_{\mathbf{e}}^{2} .
$$

If $F \in L_{\circledast \text { (e) }}^{\infty}$ and $G \in H_{\mathbb{B}(e)}^{\infty}$, the relation $F=G^{*} G$ a.e. is equivalent to $T_{F}=T_{G}^{*} T_{G}$. These facts are more or less well known. See, for example, Brown and Halmos [5].

A function $G \in H_{\mathbb{Q}(c)}^{\infty}$ is called inner (resp. outer) if $A=T_{G}$ is $S$-inner (resp. $S$-outer). In connection with this it should be noted that a subspace $\Re$ of $H_{\mathbb{e}}^{2}$ reduces $S$ if and only if $\Re=H_{\mathbb{Q}}^{2}$ for some subspace B of $\mathbb{e}$.

For any $G \in H_{\otimes(e)}^{\infty}$, the values associated with $G$ in the disk $|z|<1$ or half-plane $y>0$ have a norm-convergent representation

$$
G(z)=\sum_{0}^{\infty} A_{j} z^{j}, \quad|z|<1,
$$

or

$$
G(z)=\sum_{0}^{\infty} A_{j}\left(\frac{z-i}{z+i}\right)^{j}, \quad y>0,
$$


for some sequence $\left(A_{j}\right)_{0}^{\infty}$ in $B(\mathcal{C})$. If we make the natural identification between $\mathcal{C}$ and $\operatorname{ker} S^{*}$, the sequence $\left(A_{j}\right)_{0}^{\infty}$ also appears in the matrix representation of $T_{G}$, namely $T_{G} \sim\left[A_{j-k}\right]$ where $A_{-j}=0$, $j=1,2,3, \cdots$. In particular $A_{0}=G(0)$ or $A_{0}=G(i)$ depending on the case.

It is already clear how to translate many of the results in $\$ 1$ into corresponding theorems for bounded operator valued functions on the circle or line. However, bounded functions do not offer the natural generality for such results. Henceforth, the functions which we treat are possibly unbounded.

2.1. Definition. (i) An operator valued function $G$ on $\Gamma$ or $R$ is said to be of class $\mathfrak{T H}^{+}$if there exists a bounded scalar valued outer function $\phi(\not \equiv)$ such that $\phi G \in H_{\Re(e)}^{\infty}$.

(ii) Operator valued inner functions are defined as before: $G$ is inner if $G \in H_{\mathbb{B}(e)}^{\infty}$ and multiplication by $G$ in $H_{\mathfrak{e}}^{2}$ is a partial isometry. In this case, the initial space of multiplication by $G$ in $H_{\mathrm{e}}^{2}$ has the form $H_{\mathfrak{B}}^{2}$ where $\Theta$ is a subspace of $\mathcal{C}$, and we write $\beta=\beta_{\text {in }}(G)$.

(iii) An operator valued function $G$ on $\Gamma$ or $R$ is called outer if $G$ is of class $\Re^{+}$, and for some (and hence any) $\phi$ chosen for $G$ as in (i), $\phi G$ is outer in the sense previously defined for functions in $H_{\mathbb{Q}(e)}^{\infty}$, i.e. $\operatorname{CL}\left((\phi G) H_{\mathfrak{e}}^{2}\right)$ reduces the standard shift operator $S$. In this case $\mathrm{CL}\left((\phi G) H_{\mathfrak{e}}^{2}\right)=H_{\mathbb{Q}}^{2}$ for a subspace $\beta$ of $\mathfrak{C}$ which is independent of the choice of $\phi$, and we write $\beta=\bigotimes_{\text {out }}(G)$.

Definitions and theorems concerning operator valued functions apply in particular to scalar valued functions (specialize to the case $\operatorname{dim} \mathfrak{e}=1$ and interpret operators on $\mathcal{C}$ as scalars). In this case our definitions of inner and outer functions are consistent with the classical definitions. In the scalar case we are permitted to think of inner and outer functions as defined on the circle $\Gamma$ or line $R$, or on the disk $|z|<1$ or half-plane $y>0$. We show that the same is true for operator valued functions of class $\mathfrak{9 l}^{+}$.

An operator valued analytic function $G(z)$ defined in the disk $|z|<1$ or half-plane $y>0$ is said to have a scalar outer majorant if there exists a scalar valued outer function $\psi(z)$ such that $|G(z)|$ $\leqq|\psi(z)|$ in the disk or half-plane.

2.2. LEMmA. (i) Let $G(z)$ be an operator valued analytic function which has a scalar outer majorant in the disk $|z|<1$ or half-plane $y>0$. Then $G(z)$ has an a.e. strong boundary value function

$$
G\left(e^{i \theta}\right)=\lim _{r / 1} G\left(r e^{i \theta}\right) \text { or } G(x)=\lim _{\nu \backslash 0} G(x+i y) .
$$

If $G(z) \not \equiv 0$ then $\log \left|G\left(e^{i \theta}\right)\right| \in L^{1}(0,2 \pi)$ or $\left(1+x^{2}\right)^{-1} \log |G(x)|$ 
$\in L^{1}(-\infty, \infty)$, and $|G(z)| \leqq|\psi(z)|$ where

$$
\psi(z)=\exp \left\{\frac{1}{2 \pi} \int_{0}^{2 \pi} \frac{e^{i \theta}+z}{e^{i \theta}-z} \log \left|G\left(e^{i \theta}\right)\right| d \theta\right\}
$$

for $|z|<1$, or

$$
\psi(z)=\exp \left\{\frac{1}{\pi i} \int_{-\infty}^{\infty} \frac{1+t z}{t-z} \frac{\log |G(t)|}{1+t^{2}} d t\right\}
$$

for $y>0$ respectively.

(ii) An operator valued function $G\left(e^{i \theta}\right)$ or $G(x)$ on $\Gamma$ or $R$ is of class $\mathfrak{x}^{+}$if and only if it is a.e. equal to the boundary value function of an operator valued analytic function $G(z)$ which has a scalar outer majorant in the disk $|z|<1$ or half-plane $y>0$ respectively.

Proof. We give the proof for the circle case only. If $G(z)$ is an operator valued analytic function with a scalar outer majorant in the $\operatorname{disk}|z|<1$, then

$$
\log \left|G\left(r e^{i \theta}\right)\right| \leqq \frac{1}{2 \pi} \int_{0}^{2 \pi} \frac{1-r^{2}}{1-2 r \cos (\theta-t)+r^{2}} \log k\left(e^{i t}\right) d t
$$

for $0 \leqq r<1,0 \leqq \theta<2 \pi$, where $k\left(e^{i t}\right) \geqq 0$ a.e. and $\log k\left(e^{i t}\right) \in L^{1}(0,2 \pi)$. Construct a scalar valued outer function $\phi(z)$ such that

$$
-\log \left|\phi\left(r e^{i \theta}\right)\right|=\frac{1}{2 \pi} \int_{0}^{2 \pi} \frac{1-r^{2}}{1-2 r \cos (\theta-t)+r^{2}} \log ^{+} k\left(e^{i t}\right) d t
$$

for $0 \leqq r<1,0 \leqq \theta<2 \pi$. Then $\phi(z)$ and $\phi(z) G(z)$ are bounded functions in the disk $|z|<1$. Since every bounded operator valued analytic function in the disk has a strong boundary value function, $G\left(e^{i \theta}\right)$ exists a.e. Clearly $G\left(e^{i \theta}\right)$ is of class $\mathfrak{X}^{+}$on $\Gamma$. We have proved the existence of (8) in (i) and the sufficiency part of (ii).

Suppose $G\left(e^{i \theta}\right)$ is any operator valued function of class $\mathfrak{N}^{+}$on $\Gamma$, and set $F_{\phi}\left(e^{i \theta}\right)=\phi\left(e^{i \theta}\right) G\left(e^{i \theta}\right)$ where $\phi\left(e^{i \theta}\right)$ is chosen for $G\left(e^{i \theta}\right)$ as in Definition 2.1. Then $F_{\phi}\left(e^{i \theta}\right) \in H_{\circledast(\mathcal{C})}^{\infty}(\Gamma)$, and if $a, b \in \mathfrak{C},|a| \leqq 1$, $|b| \leqq 1$,

$$
\begin{aligned}
\log \left|\left\langle F_{\phi}\left(r e^{i \theta}\right) a, b\right\rangle_{\mathbb{e}}\right| & \leqq \frac{1}{2 \pi} \int_{0}^{2 \pi} \frac{1-r^{2}}{1-2 r \cos (\theta-t)+r^{2}} \log \left|\left\langle F_{\phi}\left(e^{i t}\right) a, b\right\rangle_{\mathbb{e}}\right| d t \\
& \leqq \frac{1}{2 \pi} \int_{0}^{2 \pi} \frac{1-r^{2}}{1-2 r \cos (\theta-t)+r^{2}} \log \left|F_{\phi}\left(e^{i t}\right)\right| d t
\end{aligned}
$$

for $0 \leqq r<1,0 \leqq \theta<2 \pi$. So if $G\left(e^{i \theta}\right)$ does not vanish a.e., then 
$\log \left|G\left(e^{i \theta}\right)\right|=\log \left|F_{\phi}\left(e^{i \theta}\right)\right|-\log \left|\phi\left(e^{i \theta}\right)\right| \in L^{1}(0,2 \pi)$, and $G\left(e^{i \theta}\right)$ is the boundary value function of $G(z)=\phi(z)^{-1} F_{\phi}(z),|z|<1$, which has the scalar outer majorant (9). The remaining parts of the lemma follow.

Let $G$ be an operator valued function of class $\Re^{+}$on $\Gamma$ or $R$. The values associated with $G$ in the disk $|z|<1$ or half-plane $y>0$ have norm convergent representations $(6)$ or $(7)$. It is not hard to see that if $G$ is an outer function, then $\Theta_{\text {out }}(G)=\mathrm{CL}(G(0) \mathcal{C})$ or $\mathrm{CL}(G(i) \mathfrak{e})$ depending on whether $G$ is defined on $\Gamma$ or $R$. Similarly, it can be shown that if $G$ is an inner function, then $\Theta_{\text {in }}(G)=\bigvee_{|w|<1} G(w) * \mathfrak{C}$ or $\mathrm{V}_{\operatorname{Im} w>0} G(w)^{*} \mathcal{C}$ depending on the case.

A consequence of Lemma 2.2 is that if $G(z)$ is an operator valued analytic function defined for $|z|<1$ such that

$$
\sup _{0 \leqq r<1} \int_{0}^{2 \pi}\left|G\left(r e^{i \theta}\right)\right|^{p} d \theta<\infty
$$

for some $p, 0<p<\infty$, then $G(z)$ has a strong boundary value function which is of class $\mathfrak{T l}^{+}$. To see this note that $u\left(r e^{i \theta}\right)=\log \left|G\left(r e^{i \theta}\right)\right|$ is subharmonic in the unit disk, and by assumption

$$
\sup _{0 \leqq r<1} \int_{0}^{2 \pi} f\left(u\left(r e^{i \theta}\right)\right) d \theta<\infty
$$

where $f(x)=e^{p x}$ for all real $x$. By a theorem of Gårding and Hörmander $[8$, p. 95], $G(z)$ satisfies an inequality of the form (11), which is equivalent to the existence of a scalar outer majorant. The analogous halfplane result uses the condition

$$
\sup _{y>0} \int_{-\infty}^{\infty}|G(x+i y)|^{p} d x<\infty
$$

where $0<p<\infty$.

2.3. THEOREM. Let $G$ be an operator valued function of class $\mathfrak{\Re}^{+}$on $\Gamma$ or $R$. Then $G$ has a unique factorization $G=G_{\mathrm{i}} G_{\mathrm{o}}$ where $G_{0}$ is an outer function such that $G_{0}(0) \geqq 0$ or $G_{0}(i) \geqq 0$ in the circle and line cases respectively, and $G_{\mathrm{i}}$ is an inner function such that $\beta_{\text {in }}\left(G_{\mathrm{i}}\right)=\bigotimes_{\text {out }}\left(G_{\mathrm{o}}\right)$. In this case $G_{0}^{*} G_{0}=G^{*} G$ a.e. Conversely, if $G_{0}$ is any operator valued outer function and $G$ is any operator valued function of class $\Re^{+}$on $\Gamma$ or $R$ such that $G^{*} G=G_{\mathrm{o}}^{*} G_{\mathrm{o}}$ a.e., then $G=G_{\mathrm{i}} G_{\mathrm{o}}$ for some inner function $G_{\mathrm{i}}$ such that $\beta_{\text {in }}\left(G_{\mathrm{i}}\right)=\beta_{\text {out }}\left(G_{\mathrm{o}}\right)$.

Proof. We easily reduce the theorem to the case where $G \in H_{\mathbb{Q} \text { (e). }}^{\infty}$. Then the result follows from Theorem 1.11 and the discussion at the beginning of this section. 
2.4. THEOREM. Let $G_{1}$ and $G_{2}$ be operator valued outer functions on $\Gamma$ or $R$.

(i) We have $G_{1}^{*} G_{1}=G_{2}^{*} G_{2}$ a.e. if and only if $G_{2}=U G_{1}, G_{1}=U^{*} G_{2}$ a.e. where $U$ is a (constant) partially isometric operator on $\mathfrak{e}$ with initial space $\Theta_{\text {out }}\left(G_{1}\right)$ and final space $\Theta_{\text {out }}\left(G_{2}\right)$.

(ii) If $G_{1}^{*} G_{1}=G_{2}^{*} G_{2}$ a.e. and $G_{j}(0) \geqq 0$ or $G_{j}(i) \geqq 0$ in the circle and line cases respectively, $j=1,2$, then $G_{1}=G_{2}$ a.e.

Proof. Suppose $G_{1}^{*} G_{1}=G_{2}^{*} G_{2}$ a.e. Choose a bounded scalar valued outer function $\phi \not \equiv 0$ such that, for each $j=1,2, F_{j}=\phi G_{j}$ is an outer

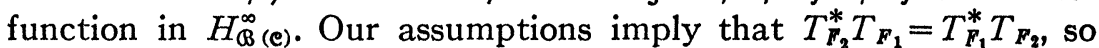
by Theorem 1.9, $T_{F_{2}}=T_{U} T_{F_{1}}, T_{F_{1}}=T_{U}^{*} T_{F_{2}}$ where $U$ is an operator valued function such that both $U$ and $U^{*}$ are inner with initial spaces $\bigotimes_{\text {out }}\left(F_{1}\right)=\bigotimes_{\text {out }}\left(G_{1}\right)$ and $\bigotimes_{\text {out }}\left(F_{2}\right)=\bigotimes_{\text {out }}\left(G_{2}\right)$ respectively. It follows that $U$ can be taken to be a constant partially isometric operator on $\mathfrak{e}$ with initial space $\Theta_{\text {out }}\left(G_{1}\right)$ and final space $\Theta_{\text {out }}\left(G_{2}\right)$. Moreover $F_{2}=U F_{1}$, $F_{1}=U^{*} F_{2}$ a.e. and so $G_{2}=U G_{1}, G_{1}=U^{*} G_{2}$ a.e. This proves the necessity of the condition in (i). The sufficiency is proved by reversing the steps in this argument. The proof of (ii) is similar, except that for (ii) we use Theorem 1.8.

If $A, B, \ldots$ are operator valued functions of class $\mathfrak{T}^{+}$on $\Gamma$ or $R$, we write $\left(A_{j}\right)_{0}^{\infty},\left(B_{j}\right)_{0}^{\infty}, \ldots$ for the coefficients in the expansions (6) and (7) for the corresponding analytic functions in the disk $|z|<1$ or half-plane $y>0$.

2.5. TheOREM. Let $C$ be an operator valued function of class $\mathfrak{x}^{+}$on $\Gamma$. Then $C$ is outer if

$$
C_{0}^{*} C_{0} \geqq A_{0}^{*} A_{0}
$$

for every operator valued function $A$ of class $\mathfrak{r}^{+}$on $\Gamma$ such that $A^{*} A$ $=C^{*} C$ a.e. Conversely, if $C$ is outer, then

$$
\sum_{0}^{n} C_{j}^{*} C_{j} \geqq \sum_{0}^{n} A_{j}^{*} A_{j}, \quad n=0,1,2, \cdots,
$$

for every operator valued function $A$ of class $9^{+}$on $\Gamma$ such that $A^{*} A$ $=C^{*} C$.

Proof. Suppose (12) holds for all $A$ as in the theorem. Choose $\phi$ for $C$ as in Definition 2.1 such that $C^{\prime}=\phi C \in H_{\mathscr{B}(e)}^{\infty}(\Gamma)$. We show that the $S$-analytic operator $T_{C^{\prime}}$ on $H_{\mathrm{e}}^{2}(\Gamma)$ is $S$-outer. Let $T_{A^{\prime}}, A^{\prime} \in H_{Q_{(0)}}^{\infty}(\Gamma)$, be any $S$-analytic operator such that $T_{A^{\prime}}^{*} T_{A^{\prime}}=T_{C^{\prime}}^{*} T_{C^{\prime}}$. If we define $A$ by $A^{\prime}=\phi A$, then $A$ is of class $\Re^{+}$and $A^{*} A=C^{*} C$ a.e. By (12) then $C_{0}^{*} C_{0} \geqq A_{0}^{*} A_{0}$, and hence 


$$
C_{0}^{\prime *} C_{0}^{\prime}=\left|\phi_{0}\right|^{2} C_{0}^{*} C_{0} \geqq\left|\phi_{0}\right|^{2} A_{0}^{*} A_{0}=A_{0}^{\prime *} A_{0}^{\prime} .
$$

It follows from Theorem 1.12 that $T_{C^{\prime}}$ is $S$-outer (see the discussion following (6) and (7)). Hence $C^{\prime}$ and $C^{\prime} / \phi$ are outer functions.

Suppose that $C$ is an outer function. Choose a sequence $\left(\phi^{(k)}\right)_{1}^{\infty}$ of

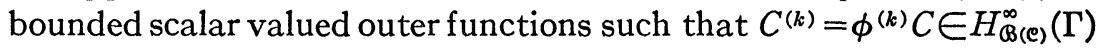
for each $k=1,2,3, \cdots$ and $\lim \phi^{(k)}(z)=1$ uniformly on any disk $|z| \leqq r, r<1$. For example, if $\phi$ is a single bounded scalar valued outer function such that $\phi C \in H_{\mathbb{Q}(\mathcal{e})}^{\infty}(\Gamma)$, we could choose

$$
\phi^{(k)}(z)=\exp \left\{\frac{1}{2 \pi} \int_{0}^{2 \pi} \frac{e^{i \theta}+z}{e^{i \theta}-z} \log \left[\min \left(k\left|\phi\left(e^{i \theta}\right)\right|, 1\right)\right] d \theta\right\},
$$

$|z|<1, k=1,2,3, \cdots$. If $\gamma_{0}^{(k)}, \gamma_{1}^{(k)}, \gamma_{2}^{(k)}, \ldots$ are the Taylor coeffcients of $1 / \phi^{(k)}$, then

$$
\begin{aligned}
\lim _{k \rightarrow \infty} \gamma_{j}^{(k)} & =1, & j=0, \\
& =0, & j>0 .
\end{aligned}
$$

But for any $n=0,1,2, \cdots, k=1,2,3, \cdots$,

$$
\sum_{j=0}^{n} C_{j}^{(k) *} C_{j}^{(k)} \geqq \sum_{j=0}^{n} A_{j}^{(k) *} A_{j}^{(k)}
$$

by Theorem 1.15 (again, see the discussion following (6) and (7)). We now deduce (13) by letting $k \rightarrow \infty$.

The continuous analogue of Theorem 2.5 is due to Robinson [23] in the case where $\operatorname{dim} \mathfrak{e}=1$.

2.6. ThEOREM. Let $C$ be an operator valued function of class $\mathfrak{r}^{+}$on $R$ such that $C(x) c \in H_{\mathfrak{e}}^{2}(R)$ for each $c \in \mathcal{C}$. Then $C$ is outer if and only if

$$
\int_{0}^{t}|v(s)|^{2} d s \geqq \int_{0}^{t}|u(s)|^{2} d s
$$

for all $t>0$, whenever $u, v$ are vector valued functions such that

$$
\left\{\begin{array}{l}
C(x) c \\
A(x) c
\end{array}\right\}=\frac{1}{(2 \pi)^{1 / 2}} \int_{0}^{\infty} e^{i x s}\left\{\begin{array}{l}
v(s) \\
u(s)
\end{array}\right\} d s
$$

for some operator valued function $A$ of class $\Re^{+}$on $R$ such that $A^{*} A$ $=C^{*} C$ a.e. and some $c \in \mathcal{C}$.

Proof. Let $\mathscr{W}=\left\{V^{t}\right\}_{t>0}$, where for each $t>0, V^{t}$ is the operator "multiplication by $e^{i t x}$ " in $H_{\mathrm{e}}^{2}(R)$. Every $f \in H_{\mathrm{e}}^{2}(R)$ has a representation 


$$
f(x)=\frac{1}{(2 \pi)^{1 / 2}} \int_{0}^{\infty} e^{i x s} g(s) d s
$$

where $g \in L_{\mathfrak{e}}^{2}(0, \infty)$, and conversely each such $g$ determines an $f \in H_{\mathfrak{e}}^{2}(R)$ by this formula. If $f$ and $g$ are so related, then

$$
\left\|V^{t * f}\right\|^{2}=\int_{t}^{\infty}|g(s)|^{2} d s
$$

for all $t>0$. By the discussion following Lemma 1.14, the weakly closed linear span of $W$ and the identity operator contains the standard shift operator $S$.

Suppose now that $C$ is an outer function. Let $A$ be any operator valued function of class $\Re^{+}$on $R$ such that $A^{*} A=C^{*} C$ a.e. By Theorem 2.3, $A=B C$ where $B$ is an inner function such that $B_{\text {in }}(B)$ $=\beta_{\text {out }}(C)$. By assumption $C(x) c \in H_{\mathfrak{e}}^{2}(R)$, and hence $A(x) c \in H_{\mathfrak{e}}^{2}(R)$ for each $c \in \mathcal{C}$. It is easy to see that $C(x) c$ is in the initial space of multiplication by $B$ in $H_{\mathfrak{e}}^{2}(R)$ for any $c \in \mathcal{C}$. So, by Lemma 1.14 (i),

$$
\left\|V^{t *} C c\right\| \leqq\left\|V^{t *} A c\right\|
$$

for all $t>0, c \in \mathcal{C}$. We get (14) by combining this with (16).

Conversely suppose that (14) holds whenever $u, v$ have the form (15). We use the inequality only for a special choice of $A$. Namely, choose $A$ outer such that $C=B A$ where $B$ is an inner function such that $\beta_{\text {in }}(B)=\beta_{\text {out }}(A)$. Such an $A$ exists by Theorem 2.3. Since $A$ is outer, we can apply what we just proved with the roles of $A$ and $C$ interchanged, and this shows that equality holds in (14), or equivalently

$$
\left\|V^{* *} A c\right\|=\left\|V^{* *} B A c\right\|
$$

for all $t>0$ and $c \in \mathbb{C}$. Let $\mathcal{G}$ be the set of elements of $H_{\mathfrak{e}}^{2}(R)$ of the form $A c, c \in \mathcal{C}$. With this choice of $G$ and $W$ as above we apply Lemma 1.14 (ii) to the $S$-inner operator "multiplication by $B$ " in $H_{\mathrm{e}}^{2}(R)$. It follows from the lemma that $B^{*}$ is also an inner function. Hence $B$ can be taken to be a constant partially isometric operator on $\mathrm{C}$. Finally, since $A$ is an outer function, so is $C$.

Singularities of operator valued analytic functions are classified in exactly the same way as for scalar valued analytic functions. An operator valued function $F(z)$ is said to be meromorphic in a region $\Omega$ if $F(z)$ is defined and analytic except at certain isolated points which are poles. An operator valued function $F(z)$ which is meromorphic in the extended complex plane is called a rational function. Every opera- 
tor valued rational function has the form $P(z) / q(z)$ where $P(z)$ $=A_{0}+A_{1} z+\cdots+A_{m} z^{m}$ is a polynomial with operator coefficients and $q(z)=b_{0}+b_{1} z+\cdots+b_{n} z^{n}$ is a polynomial with scalar coeffcients, $q(z) \not \equiv$, and conversely.

2.7. Definition. Let $u, v$ be nonzero scalar valued functions of class $\Re^{+}$on $\Gamma$ or $R$. An operator valued function $F$ on $\Gamma$ or $R$ is said to be of class $\Re(u, v)$ if $u F$ and $v F^{*}$ are of class $\Re^{+}$.

If $u=u_{\mathrm{i}} u_{\mathrm{o}}, v=v_{\mathrm{i}} v_{\mathrm{o}}$ are inner-outer factorizations of $u, v$, then a function $F$ is of class $\operatorname{Tr}(u, v)$ if and only if $F$ is of class $\operatorname{Tr}\left(u_{\mathrm{i}}, v_{\mathrm{i}}\right)$. In other words the class $\mathfrak{T}(u, v)$ is independent of the outer factors in $u, v$.

We show that functions of class $\mathfrak{T}(u, v)$ can be characterized as two-sided boundary value functions of certain kinds of meromorphic functions defined off $\Gamma$ or $R$. In particular, we can again speak of function values off $\Gamma$ or $R$.

If $F(z)$ is an operator valued function defined in a region $\Omega$ of the complex plane, we write $\tilde{F}(z)$ for one of the reflected functions $\tilde{F}(z)=F(\bar{z})^{*}$ in $\tilde{\Omega}=\{z: \bar{z} \in \Omega\}$ or $\tilde{F}(z)=F(1 / \bar{z})^{*}$ in $\tilde{\Omega}=\{z: 1 / \bar{z} \in \Omega\}$, depending on context.

2.8. THEOREM. Let $u, v$ be nonzero scalar valued functions of class $\mathfrak{\Re}^{+}$ on $\Gamma$ or $R$.

(i) Let $F(z)$ be an operator valued function which is defined and meromorphic separately for $|z|<1,|z|>1$ or for $y>0, y<0$. Suppose that $u(z) F(z), v(z) \widetilde{F}(z)$ are analytic and have scalar outer majorants in the disk $|z|<1$ or half-plane $y>0$. Suppose that $F\left(r e^{i \theta}\right)$ has the same strong limit $F\left(e^{i \theta}\right)$ a.e. as $r \nearrow 1$ and $r \searrow 1$, respectively $F(x+i y)$ has the same strong limit $F(x)$ a.e. as $y \searrow 0$ and $y \nearrow 0$. Then the boundary value function is of class $\mathfrak{T}(u, v)$ on $\Gamma$ or $R$, and conversely every operator valued function of class $\Re(u, v)$ on $\Gamma$ or $R$ arises in this way.

(ii) In the situation of (i), if the boundary value function is essentially bounded on an open arc on $\Gamma$ or interval on $R$, and if $u$ and $v$ are analytic across the arc or interval, then the meromorphic functions are analytic continuations of each other across the arc or interval.

In [14] Kriete proves that a square-integrable scalar valued function of class $\mathscr{T}(u, v)$ has an integral representation analogous to the Paley-Wiener representation theorem for entire functions of exponential type which are square integrable on the real axis. ${ }^{1}$

Proof. We give this proof for the line case only.

(i) Suppose $F(z)$ is meromorphic for $y>0, y<0$, and the assump-

${ }^{1}$ Kriete discovered this result in the academic year 1966-67 and circulated it privately, before the authors began work on the present manuscript. 
tions in (i) hold. By Lemma 2.2, $u(x) F(x)$ and $v(x) F(x)^{*}$ are functions of class $\mathfrak{x}^{+}$on $R$, so we immediately deduce that $F(x)$ is of class $\mathscr{T}(u, v)$ on $R$. Conversely, if $F(x)$ is a given function of class $\mathscr{T}(u, v)$ on $R$, then by Lemma $2.2, u(x) F(x)$ and $v(x) F(x)^{*}$ are a.e. strong boundary value functions of operator valued analytic functions $F_{+}(z)$ and $F_{-}(z)$ which are defined and have scalar outer majorants in the half-plane $y>0$. The function

$$
\begin{aligned}
F(z) & =F_{+}(z) / u(z), & & y>0, \\
& =\tilde{F}_{-}(z) / \tilde{v}(z), & & y<0,
\end{aligned}
$$

is meromorphic separately for $y>0, y<0$, it satisfies the conditions in (i), and it has $F(x)$ as an a.e. strong two-sided boundary value function. In fact, we get at once that $F(x)=\lim F(x+i y)$ a.e. as $y \searrow 0$ in the strong operator topology, and as $y \nearrow 0$ in the weak operator topology. But weak convergence can be improved to strong convergence here by the analogue of Lemma 2.2 for the lower half-plane.

(ii) Using notation as above, we assume now that $u(z)$ and $v(z)$ have analytic continuations across an interval $(a, b)$ and that $F(x)$ is essentially bounded on $(a, b)$. We may assume without loss of generality that the continuations of $u(z)$ and $v(z)$ have no zeros on $(a, b)$. For we can contract the interval $(a, b)$ slightly so that only a finite number of zeros are present, and these can be removed by dividing out a suitable outer factor (see the remark following Definition 2.7). Alternately, it can be shown that the inner parts of $u$ and $v$ are analytic and nonzero in a neighborhood of $(a, b)$. Then, using scalar majorants for $F_{+}(z)$ and $F_{-}(z)$ of the form (10) and the fact that $F(x)$ is essentially bounded on $(a, b)$, we can reduce to the case where $F(z)$ is bounded on and inside some rectangle $\rho: x=a$, $x=b, y=-\delta, y=\delta(\delta>0)$, ignoring points on the real axis. At worst we will have to contract the interval $(a, b)$ slightly once more. But now straightforward arguments will show that

$$
G(z)=\frac{1}{2 \pi i} \oint_{\rho} \frac{F(t)}{t-z} d t
$$

is analytic in the interior of $\rho$ and is the required continuation of $F(z)$ across $(a, b)$.

An operator valued function $F$ on $\Gamma$ or $R$ is in $H_{\circledast \text { (e) }}^{\infty}$ if and only if $F$ is essentially bounded and for each $c \in \mathcal{C}$ the scalar valued function $F_{c}(\cdot)=\langle F(\cdot) c, c\rangle_{e}$ is in $H^{\infty}$ on $\Gamma$ or $R$. This follows from elementary properties of Fourier series. It is convenient to have an analogous characterization of the classes $\mathfrak{T}^{+}$and $\mathfrak{T}(u, v)$. 
2.9. THEOREM. Let $F$ be an operator valued function on $\Gamma$ or $R$. Then $F$ is of class $\mathfrak{X}^{+}$if and only if for each $c \in \mathcal{e}$ the scalar valued function $F_{\mathrm{c}}$ is of class $\mathfrak{I C}^{+}$and

$$
\int_{0}^{2 \pi} \log ^{+}\left|F\left(e^{i \theta}\right)\right| d \theta<\infty \text { or } \int_{-\infty}^{\infty}\left(1+x^{2}\right)^{-1} \log ^{+}|F(x)| d x<\infty .
$$

If $u, v$ are nonzero scalar valued functions of class $\mathfrak{\eta}^{+}$then $F$ is of class $\mathfrak{M}(u, v)$ if and only if $F_{\mathrm{c}}$ is of class $\mathfrak{M}(u, v)$ for each $c \in \mathcal{C}$ and $(17)$ holds.

Proof. Suppose that $F_{c}$ is of class $\mathfrak{I}^{+}$for all $c \in \mathcal{C}$ and (17) holds. By (17) there is a scalar valued outer function $\phi$ such that $|\phi|^{-1}$ $=\max (|F|, 1)$. Then $\phi$ and $G=\phi F$ are bounded functions, and $G_{0}$ $=\phi F_{\mathrm{c}}$ is of class $\mathfrak{T}^{+}$for all $c \in \mathrm{e}$. Hence $G_{c}$ is in $H^{\infty}$ for all $c \in \mathrm{e}$, so $G \in H_{\mathscr{Q}(e)}^{\infty}$. Therefore $F$ is of class $\mathfrak{I}^{+}$. We omit the easy proof of the converse statement. The second assertion follows directly from the first.

3. The factorization problem for nonnegative operator valued functions. Let $F$ be a nonnegative operator valued function defined on the the circle $\Gamma$ or the line $R$. We call $F$ factorable if $F=G^{*} G$ a.e. for some operator valued function $G$ of class $\Re^{+}$on $\Gamma$ or $R$. If $F$ is factorable, then, by Theorems 2.3 and 2.4, $F=G^{*} G$ a.e. for an essentially unique outer function $G$, or a unique outer function $G$ such that $G(0) \geqq 0$ or $G(i) \geqq 0$ in the circle and line cases respectively. Here, of course, we treat functions which are equal a.e. as being identical, and "essential uniqueness" refers to constant partially isometric left factors as in Theorem 2.4.

In the special case where $F \in L_{\mathscr{Q}(\mathcal{C})}^{\infty}, F$ is factorable if and only if the induced $S$-Toeplitz operator $T_{F}$ on $H_{\mathfrak{e}}^{2}$ is factorable in the sense of Definition 1.5. Therefore the results in $\$ 1$ can be made to yield sufficient conditions for $F$ to be factorable.

Suppose, for example, that $F$ is a bounded nonnegative operator valued function on $\Gamma$ or $R$ which is of class $\Pi(v, v)$ for some nonzero scalar valued function $v$ of class $\mathfrak{g}^{+}$. By Theorem 1.16, $F$ is factorable, and moreover if $G$ is an outer function such that $F=G^{*} G$ a.e. then $G$ is of class $\Re(1, v)$. This result is already sufficient for a number of interesting applications. But in fact the assumption that $F$ is bounded is redundant.

3.1. THEOREM. Let $F$ be a nonnegative operator valued function defined on $\Gamma$ or $R$ which is of class $\operatorname{Tr}(v, v)$ for some nonzero scalar valued function $v$ of class $\mathrm{g}^{+}$. Then $F$ is factorable, and moreover if $G$ is any outer function such that $F=G^{*} G$ a.e., then $G$ is of class $\operatorname{mi}(1, v)$. 
PROOF. We give the proof in the circle case. We can assume without loss of generality that $v$ is an inner function. By assumption $v F$ is of class $\mathfrak{N}^{+}$, so there exists a bounded scalar valued outer function $\phi(\not \equiv 0)$ such that $\phi v F \in H_{\mathfrak{B}(\mathcal{C})}^{\infty}(\Gamma)$. The function $K=\bar{\phi} F \phi$ is bounded and it induces an $S$-Toeplitz operator $T_{K}$ on $H_{\mathfrak{e}}^{2}(\Gamma)$. We show that $T_{K}$ is factorable as an $S$-Toeplitz operator.

By Theorem 1.7 it is enough to show that $\lim I_{n}(c)=0$ as $n \rightarrow \infty$ for each $c \in \mathfrak{e}$, where

$$
I_{n}(c)=\sup \left\{\left|\left\langle T_{K} c, S^{n} f\right\rangle\right|: f \in H^{2} \mathrm{e}(\Gamma),\left\langle T_{K} f, f\right\rangle=1\right\}
$$

for all $c \in \mathcal{C}$ and $n=0,1,2, \cdots$. Here $\mathcal{C}$ is regarded as a subspace of $H_{\mathrm{e}}^{2}(\Gamma)$ in the natural way. Let $\sigma$ be normalized Lebesgue measure on $\Gamma$; let $\chi\left(e^{i \theta}\right)=e^{i \theta}, e^{i \theta} \in \Gamma$; and let $P$ be the projection mapping $L_{\mathfrak{e}}^{2}(\Gamma)$ on to $H_{\mathfrak{e}}^{2}(\Gamma)$. Recall that $\phi v F \in H_{\mathbb{B}(\mathcal{e})}^{\infty}(\Gamma)$ and $|v|=1$ a.e. Consider $c \in \mathfrak{e}, f \in H_{\mathfrak{e}}^{2}(\Gamma)$, and estimate as follows:

$$
\begin{aligned}
\left|\left\langle T_{K} c, S^{n} f\right\rangle\right| & =\left|\int_{\Gamma}\left\langle K c, \chi^{n} f\right\rangle_{e} d \sigma\right| \\
& =\left|\int_{\Gamma}\left\langle\frac{\phi}{\phi^{*}} v c, \chi^{n} \phi^{2} v F f\right\rangle_{e} d \sigma\right| \\
& =\left|\int_{\Gamma}\left\langle P \chi^{-n} P \frac{\phi}{\phi^{*}} v c, v\left(F^{1 / 2} \phi\right)^{2} f\right\rangle_{e} d \sigma\right| \\
& =\left|\int_{\Gamma}\left\langle F^{1 / 2} \phi^{*} P \chi^{-n} P \frac{\phi}{\phi^{*}} v c, v F^{1 / 2} \phi f\right\rangle_{e} d \sigma\right| \\
& \leqq\left(\int_{\Gamma}\left|F^{1 / 2} \phi^{*} P \chi^{-n} P \frac{\phi}{\phi^{*}} v c\right|^{2} d \sigma\right)^{1 / 2}\left\langle T_{K} f, f\right\rangle
\end{aligned}
$$

for any $n=0,1,2, \cdots$. The inner products and norms appearing inside an integral apply to function values and, of course, are taken pointwise. Since $F^{1 / 2} \phi^{*}$ is a bounded function by the choice of $\phi$, we get

$$
I_{n}(c) \leqq \text { const. }\left(\int_{\Gamma}\left|P \chi^{-n} P \frac{\phi}{\phi^{*}} v c\right|^{2} d \sigma\right)^{1 / 2}=\text { const. }\left\|S^{*_{n}} g c\right\|
$$

for all $c \in \mathcal{C}$ and $n=0,1,2, \cdots$, where $g_{c}=P\left(\phi / \phi^{*}\right) v c$. Since $S$ is a shift operator, $\lim I_{n}(c)=0$ as $n \rightarrow \infty$ for all $c \in \mathcal{C}$, so $T_{K}$ is factorable by Theorem 1.7 .

It follows that $K$ is factorable, and hence so is $F$. In fact, $K=M^{*} M$ a.e. where $M \in H_{\mathbb{B}(\mathcal{C})}^{\infty}(\Gamma)$ and so $F=G^{*} G$ a.e. where $G=\phi^{-1} M$ is of class $\mathfrak{T}^{+}$. 
If $G$ is an outer function such that $F=G^{*} G$ a.e. then $M=\phi G$ is an outer function in $H_{\Theta_{(\mathcal{C})}}^{\infty}(\Gamma)$ such that $M^{*} M=K$ a.e. We show that the function $W=\phi v G^{*}$ is in $H_{\mathbb{B}(\mathrm{e})}^{\infty}(\Gamma)$, thus proving that $v G^{*}$ is of class $\Re^{+}$and hence $G$ is of class $\operatorname{Tl}(1, v)$. Clearly $W=v M^{*} \phi / \phi^{*}$ is bounded, and, by the choice of $\phi$,

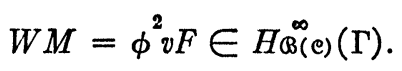

Therefore $W \mathrm{CL}\left(M H_{\mathrm{e}}^{2}(\Gamma)\right) \subseteq \mathrm{CL}\left(H_{\mathrm{e}}^{2}(\Gamma)\right)$. Since $M$ is a bounded outer function, if $g \in H_{\mathfrak{e}}^{2}(\Gamma) \ominus \mathrm{CL}\left(M H_{\mathfrak{e}}^{2}(\Gamma)\right)$ then $S^{j} g \in H_{\mathfrak{e}}^{2}(\Gamma) \ominus \operatorname{CL}\left(M H_{\mathfrak{e}}^{2}(\Gamma)\right)$ for all $j=0,1,2, \cdots$.

Hence

$$
\int_{\Gamma}\left\langle M^{*} g, \chi^{-j} u\right\rangle d \sigma=\int_{\Gamma}\left\langle\chi^{j} g, M u\right\rangle d \sigma=0
$$

for all $u \in H_{\mathfrak{e}}^{2}(\Gamma), j=0,1,2, \cdots$. It follows that $M^{*} g=0$ a.e. so $W g=0$ a.e. But then $W H_{\mathfrak{e}}^{2}(\Gamma) \subseteq H_{\mathfrak{e}}^{2}(\Gamma)$ so $W \in H_{\overparen{B}(\mathcal{e})}^{\infty}(\Gamma)$ and the proof is complete.

Special choices of $v$ in Theorem 3.1 lead to factorization theorems for polynomials, rational functions, and entire functions of exponential type. However, for polynomials and rational functions (bounded or unbounded on $\Gamma$ or $R$ ) we do not need the full strength of Theorem 3.1. In these cases it would be possible to argue directly from Theorem 1.16 .

The operator generalization of the Fejér-Riesz theorem is due to Rosenblum [25], Rosenblatt [24], and Gohberg [9]. See also Helson [10].

3.2. Theorem. Let $F\left(e^{i \theta}\right)=\sum_{-n}^{n} A_{j} e^{i j \theta}$ be a trigonometric polynomial whose coefficients are operators on $\mathcal{C}$ and which is nonnegative on $\Gamma$. Then $F=G^{*} G$ where $G$ is an outer function on $\Gamma$ of the form $G\left(e^{i \theta}\right)$ $=\sum_{0}^{n} C_{j} e^{i j \theta}$ for some operators $C_{0}, \cdots, C_{n}$ on $\mathfrak{e}$.

Proof. Let $u\left(e^{i \theta}\right)=e^{i m \theta}, v\left(e^{i \theta}\right)=e^{i n \theta}$ on $\Gamma$ where $m$ and $n$ are nonnegative integers. A bounded weakly measurable operator valued function $K$ on $\Gamma$ is of class $\operatorname{Tr}(u, v)$ if and only if $u K, v K^{*} \in H_{\circledast(\odot)}^{\infty}(\Gamma)$, or

$$
\int_{0}^{2 \pi}\left\langle K\left(e^{i \theta}\right) a, b\right\rangle e^{i j \theta} d \theta=0
$$

whenever $a, b \in \mathcal{C}$ and either $j>m$ or $j<-n$, or $K\left(e^{i \theta}\right)=\sum_{-m}^{n} B_{j} e^{i j \theta}$ for some operators $B_{-m}, \cdots, B_{n}$ on $\mathcal{C}$. Thus the theorem follows from either Theorem 3.1 or Theorem 1.16 .

3.3. Theorem. Let $P(x)=\sum_{0}^{2 n} P_{j} x^{j}$ be a polynomial whose coeff- 
cients are operators on $\mathrm{C}$ and which is nonnegative on $R$. Then $P=Q^{*} Q$ where $Q$ is an outer function on $R$ of the form $Q(x)=\sum_{0}^{n} Q_{j} x^{j}$ for some operators $Q_{0}, \cdots, Q_{n}$ on $\mathfrak{C}$.

Proof. The function $F(z)=\left(z^{2}+1\right)^{-n} P(z)$ is meromorphic in the complex plane. Setting $v(z)=[(z-i) /(z+i)]^{n}$ we see that $v(z) F(z)$ and $v(z) \widetilde{F}(z)$ are analytic and bounded in the half-plane $y>0$. By Theorem 2.8, $F(x)$ is of class $\pi(v, v)$ on $R$. By Theorem 3.1 or Theorem 1.16, $F(x)=G(x) * G(x)$ on $R$ where $G(x)$ is an outer function on $R$ of class $\operatorname{Ir}(1, v)$. By Theorem $2.8, G(x)$ is the restriction to the real axis of a meromorphic function $G(z)$, defined in the complex plane and analytic at each real point, such that $G(z)$ and $v(z) \tilde{G}(z)$ are analytic and have scalar outer majorants for $y>0$. Since $G(x)$ and $v(x) \tilde{G}(x)$ are bounded on $R$, actually $G(z)$ and $v(z) \tilde{G}(z)$ are bounded for $y>0$ by Lemma 2.2 . Thus, considering the upper and lower half-planes separately, we see that

$$
\left|(z+i)^{n} G(z)\right| \leq M(|z|+1)^{n}
$$

for all complex $z$ and some $M>0$. By Cauchy's estimates, $Q(z)$ $=(z+i)^{n} G(z)$ is a polynomial of degree at most $n$. Since $G(x)$ is an outer function on $R$, so is $Q(x)$. By construction $P(x)=Q(x)^{*} Q(x)$ on $R$ and the theorem follows.

In $\$ 2$ and the present section we have the standing assumption that $\mathfrak{C}$ is a separable Hilbert space. Actually Theorems 3.2 and 3.3 are valid for nonseparable spaces $\mathcal{C}$ as well. Basically all that is needed to extend the proofs is to replace $H_{\mathfrak{e}}^{2}(\Gamma)$ by a more general Hilbert space of square summable sequences or formal power series. If we then argue directly from Theorem 1.16, we obtain a proof of Theorem 3.2 without the separability assumption. But then Theorem 3.3 can be deduced from Theorem 3.2 by a transformation of the independent variables. We chose a direct proof of Theorem 3.3 above to convey some feeling for the classes $\operatorname{\Re r}(u, v)$.

3.4. THEOREM. Let $F(z)$ be an operator valued rational function which is either nonnegative at each point $e^{i \theta} \in \Gamma$ which is not a pole, or nonegative at each point $x \in R$ which is not a pole. Then $F(z)=\widetilde{G}(z) G(z)$ where $G(z)$ is an operator valued rational function such that the restriction of $G(z)$ to the circle $\Gamma$ or line $R$ is an outer function. In particular, this $G(z)$ is analytic in the disk $|z|<1$ or half-plane $y>0$ respectively.

Proof. We may choose a nonzero scalar polynomial $q(z)$ such that the restriction of $q(z)$ to $\Gamma$ or $R$ is an outer function and $P(z)$ $=\tilde{q}(z) q(z) F(z)$ is a polynomial in $1 / z$ and $z$, respectively a polynomial in $z$, with nonnegative values on $\Gamma$ or $R$. Factor $P\left(e^{i \theta}\right)=Q\left(e^{i \theta}\right)^{*} Q\left(e^{i \theta}\right)$ or $P(x)=Q(x)^{*} Q(x)$ on $\Gamma$ or $R$ as in Theorem 3.2 or Theorem 3.3. 
The required factorization is then obtained with $G(z)=Q(z) / q(z)$.

Let $f$ be a scalar valued entire function. We say that $f$ is of exponential type if there is a real number $\tau$ such that $|f(z)| \leqq K e^{\tau|z|}$ for all $z$ in the complex plane and some $K>0$. In this case the exact type of $f$ is defined to be the infimum $\tau_{f}$ of all such numbers $\tau$. By Liouville's theorem $\tau_{f} \geqq 0$ except when $f \equiv 0$, in which case $\tau_{f}=-\infty$. We say that $f$ is of exponential type $\tau$, where $\tau$ is a real number if $f$ is of exponential type and $\tau_{f} \leqq \tau$. For any entire function $f, \tilde{f}$ is the entire function such that $\bar{f}(z)=\bar{f}(\bar{z})$. By a theorem of M. G. Krein [13], if $f$ is an entire function the following are equivalent:

(i) the restrictions of $f$ and $\tilde{f}$ to the upper half-plane $y>0$ are of bounded type in the half-plane.

(ii) $f$ is of exponential type and

$$
\int_{-\infty}^{\infty}\left(1+x^{2}\right)^{-1} \log ^{+}|f(x)| d x<\infty .
$$

See also de Branges [3]. If $f$ satisfies (i) and (ii) and is not identically zero, Nevanlinna's factorization gives

$$
\begin{aligned}
& f(z)=C_{+} B_{+}(z) e^{-i \tau+z} \exp \left\{\frac{1}{\pi i} \int_{-\infty}^{\infty} \frac{1+t z}{t-z} \frac{\log |f(t)|}{1+t^{2}} d t\right\}, \\
& f(z)=C_{-} B_{-}(z) e^{-i \tau_{-} z} \exp \left\{\frac{1}{\pi i} \int_{-\infty}^{\infty} \frac{1+t z}{t-z} \frac{\log |f(t)|}{1+t^{2}} d t\right\}
\end{aligned}
$$

for $y>0$, where $C_{+}, C_{-}$are constants of modulus $1, \tau_{+}, \tau_{-}$are real numbers, $B_{+}(z), B_{-}(z)$ are Blaschke products, and the integrals converge absolutely. The numbers $\tau_{+}, \tau_{-}$are called the mean types of $f$ and $\tilde{f}$ respectively (with respect to the upper half-plane). One of them may be negative, but $\tau_{+}+\tau_{-} \geqq 0$ and $\tau_{f}=\max \left(\tau_{+}, \tau_{-}\right),\left|\tau_{ \pm}\right| \leqq \tau_{f}$, where $\tau_{f}$ is the exact type of $f$. If $f \equiv 0$ we set $\tau_{+}=\tau_{-}=-\infty$. In general

$$
\tau_{ \pm}=\limsup _{y \rightarrow \infty} y^{-1} \log |f( \pm i y)| \text {. }
$$

See Boas [2], de Branges [3]. An entire function $f$ is said to be of Krěn class, more precisely of class $\mathfrak{K}\left(\tau_{1}, \tau_{2}\right)$ where $\tau_{1}, \tau_{2} \geqq 0$, if $f$ satisfies the equivalent conditions (i) and (ii), and $\tau_{+} \leqq \tau_{1}, \tau_{-} \leqq \tau_{2}$ where $\tau_{+}, \tau_{-}$are defined for $f$ as above.

We extend these concepts to operator valued functions. Recall that for any operator valued function $F$ and any $c \in \mathcal{e}, F_{c}$ is the scalar valued function defined by $F_{c}(\cdot)=\langle F(\cdot) c, c\rangle_{\mathfrak{e}}$. An operator valued entire function $F$ is said to be of exponential type $\tau$ if for each $c \in \mathfrak{C}$ the scalar valued entire function $F_{c}$ is of exponential type $\tau$. An opera- 
tor valued entire function $F$ is said to be of class $\nVdash\left(\tau_{1}, \tau_{2}\right), \tau_{1} \geqq 0$, $\tau_{2} \geqq 0$, if for each $c \in \mathcal{C}$ the scalar valued entire function $F_{c}$ is of class $\Re\left(\tau_{1}, \tau_{2}\right)$, and moreover

$$
\int_{-\infty}^{\infty}\left(1+x^{2}\right)^{-1} \log ^{+}|F(x)| d x<\infty .
$$

3.5. Lemma. Let $F(x)$ be a locally bounded operator valued function defined on $R$. Let $u(x)=e^{i \tau_{1} x}, v(x)=e^{i \tau_{2} x}$ on $R$ where $\tau_{1} \geqq 0, \tau_{2} \geqq 0$. Then $F(x)$ is of class $\mathscr{T}(u, v)$ if and only if $F(x)$ is the a.e. restriction to the real axis of an operator valued entire function of class $\Re\left(\tau_{1}, \tau_{2}\right)$.

Proof. Suppose that $F(x)=G(x)$ a.e. on $R$ where $G(z)$ is an operator valued entire function of class $\mathscr{K}\left(\tau_{1}, \tau_{2}\right)$. Using the Nevanlinna factorization we see at once that for each $c \in \mathcal{C}, F_{c}$ is of class $\mathfrak{M}(u, v)$. Also, by the definition of $\mathscr{K}\left(\tau_{1}, \tau_{2}\right),\left(1+x^{2}\right)^{-1} \log ^{+}|F(x)| \in L^{1}(-\infty, \infty)$. Therefore by Lemma $2.9, F$ is of class $\operatorname{Tr}(u, v)$.

Conversely assume that $F$ is of class $\operatorname{Tr}(u, v)$. Since $F$ is locally bounded and $u(z)=e^{i \tau_{1} z}, v(z)=e^{i \tau_{2} z}$ are entire functions, by Lemma 2.8, $F(x)=G(x)$ a.e. for some operator valued entire function $G(z)$ such that $e^{i r_{1} z} G(z)$ and $e^{i r_{2} z} \tilde{G}(z)$ have scalar outer majorants in the half-plane $y>0$. It follows from Kren̆n's theorem that, for each $c \in \mathcal{C}$, the scalar valued entire function $G_{c}$ is of exponential type. By (18) the mean types of $G_{c}$ and $\left(G_{c}\right)^{\sim}=(\tilde{G})_{c}$ do not exceed $\tau_{1}$ and $\tau_{2}$ respectively. In other words $G_{c}$ is of class $\Re\left(\tau_{1}, \tau_{2}\right)$ for each $c \in \mathcal{C}$. By Lemma 2.2, $\left(1+x^{2}\right)^{-1} \log ^{+}|G(x)| \in L^{1}(-\infty, \infty)$, and hence $G(z)$ is of class $\Re\left(\tau_{1}, \tau_{2}\right)$. The result follows.

We can now state an operator generalization of a classical factorization theorem for entire functions of exponential type which are nonnegative on the real axis (Boas [2, p. 125], de Branges [3, p. 34], Levin $[16$, p. 437]).

3.6. Theorem. Let $F(z)$ be an operator valued entire function of exponential type $\tau, \tau \geqq 0$, such that $F(x) \geqq 0$ for all real $x$, and

$$
\int_{-\infty}^{\infty}\left(1+x^{2}\right)^{-1} \log ^{+}|F(x)| d x<\infty .
$$

Then $F(z)=\tilde{G}(z) G(z)$ for some operator valued entire function $G(z)$ such that $e^{-(1 / 2) \text { irz }} G(z)$ is of exponential type $\frac{1}{2} \tau$ and the restriction of $G(z)$ to the real axis is an outer function.

A similar result is proved by the authors in [26]. The theorem stated here is a stronger result, and the proof which we give is considerably simpler than our previous argument. 
Proof. The function $F(z)$ is of class $\mathscr{K}(\tau, \tau)$, so by Lemma 3.5 its restriction $F(x)$ to $R$ is of class $\operatorname{Tr}(v, v)$ where $v(x)=e^{i \tau x}$. Factor $F(x)=G(x)^{*} G(x)$ a.e. as in Theorem 3.1 where $G(x)$ is outer and of class $\mathfrak{M}(1, v)$. By Lemma 3.5 we can alter $G(x)$ on a set of measure zero so that it becomes the restriction to $R$ of an operator valued entire function $G(z)$ of class $\Re(0, \tau)$. By analyticity $F(z)$ $=\widetilde{G}(z) G(z)$ for all complex $z$, and $e^{-(1 / 2) i r z} G(z)$ is of class $\mathcal{K}\left(\frac{1}{2} \tau, \frac{1}{2} \tau\right)$ and hence of exponential type $\frac{1}{2} \tau$.

Theorem 3.6 has a curious application to scalar valued entire functions.

3.7. ThEOREM. Let $f$ be a scalar valued entire function of exponential type $\tau, \tau \geqq 0$, such that $|f(x)| \leqq 1$ for all real $x$. Then $f=a b+c d$ where $a, b, c, d$ are entire functions of exponential type $\frac{1}{2} \tau$ such that $\tilde{a} a+\tilde{\tau} c$ $=\tilde{b} b+\tilde{d} d=1$ identically.

Proof. Apply Theorem 3.6 to the matrix valued entire function $F=\left[\begin{array}{ll}1 & f \\ \tilde{j} & 1\end{array}\right]$. Write $e^{-(1 / 2) i r z} G=\left[\begin{array}{ll}\tilde{a} & b \\ z & d\end{array}\right]$ and compare matrix entries in the relation $F=\widetilde{G} G$.

We return again to the general factorization problem for nonnegative operator valued functions on the circle or line. For functions which have invertible values a.e. there is a simple sufficient condition for factorability (Lowdenslager [17], Devinatz [6], Douglas [7]).

3.8. THEOREM. Let $F$ be a weakly measurable nonnegative operator valued function which has invertible values a.e. on $\Gamma$ or $R$. Suppose that either

$$
\log ^{+}\left|F\left(e^{i \theta}\right)\right| \text { and } \log ^{+}\left|F\left(e^{i \theta}\right)^{-1}\right| \in L^{1}(0,2 \pi)
$$

or

$\left(1+x^{2}\right)^{-1} \log ^{+}|F(x)|$ and $\left(1+x^{2}\right)^{-1} \log ^{+}\left|F(x)^{-1}\right| \in L^{1}(-\infty, \infty)$.

Then $F$ is factorable, i.e. $F=G^{*} G$ a.e. for some operator valued function $G$ of class $\mathfrak{Y}^{+}$on $\Gamma$ or $R$.

Proof. We reduce to the case where $F$ is bounded. In fact, consider $F_{1}=F / f$ where $f(\cdot)=\max (1,|F(\cdot)|)$. Our assumptions imply that $f$ is a factorable scalar valued function, so $F$ and $F_{1}$ are simultaneously factorable or not. Since $0 \leqq F_{1} \leqq I$ on $\Gamma$ or $R$, we may assume that $0 \leqq F \leqq I$ on $\Gamma$ or $R$. But now $F \geqq m I$ on $\Gamma$ or $R$ where $m(\cdot)=\left|F(\cdot)^{-1}\right|^{-1}$ is a factorable scalar valued function, say $m=|\psi|^{2}$ where $\psi$ is a bounded scalar valued outer function on $\Gamma$ or $R$. The proof can now be completed by showing that the hypotheses of Theorem 1.18 are satisfied with $\mathfrak{H C}=H_{\mathfrak{e}}^{2}, T_{1}=T_{m I}$, and $T_{2}=T_{F}$. If $\left(f_{n}\right)_{1}^{\infty}$ is a sequence in 
$H_{\mathrm{e}}^{2}$ such that

$$
\lim _{n, k \rightarrow \infty}\left\langle T_{2}\left(f_{n}-f_{k}\right), f_{n}-f_{k}\right\rangle=0 \text { and } \lim _{n \rightarrow \infty}\left\langle T_{1} f_{n}, f_{n}\right\rangle=0,
$$

then $\left(F^{1 / 2} f_{n}\right)_{1}^{\infty}$ is a Cauchy sequence in $L_{\mathrm{e}}^{2}$ and $\left(\psi f_{n}\right)_{1}^{\infty}$ converges to 0 in the metric of $L_{\mathrm{e}}^{2}$. But then $\psi f_{n_{j}} \rightarrow 0$ a.e. for a suitable subsequence, and hence $f_{n_{j}} \rightarrow 0$ a.e. and $F^{1 / 2} f_{n_{j}} \rightarrow 0$ a.e. (in the metric of $\mathcal{C}$ ). It follows that $F^{1 / 2} f_{n} \rightarrow 0$ in the metric of $L_{\mathrm{e}}^{2}$ and $\lim _{n \rightarrow \infty}\left\langle T_{2} f_{n}, f_{n}\right\rangle=0$. By Theorem 1.18, $T_{2}=T_{F}$ is factorable as an $S$-Toeplitz operator and therefore $F$ is factorable.

\section{REFERENCES}

1. N. I. Ahiezer, On the theory of entire functions of finite degree, Dokl. Akad. Nauk SSSR 63 (1948), 475-478. (Russian) MR 10, 289.

2. R. P. Boas, Jr. Entire functions, Academic Press, New York, 1954. MR 16, 914.

3. L. de Branges, Hilbert spaces of entire functions, Prentice-Hall, Englewood Cliffs, New Jersey, 1968. MR 37 \#4590.

4. L. de Branges and J. Rovnyak, "Appendix on square summable power series," in Canonical models in quantum scattering theory, Perturbation Theory and its Applications in Quantum Mechanics, Wiley, New York, 1966, pp. 347-392.

5. A. Brown and P. R. Halmos, Algebraic properties of Toeplitz operators, J. Reine Angew. Math. 213 (1963/64), 89-102. MR 28 \#3350; MR 30 p. 1205.

6. A. Devinatz, The factorization of operator valued functions, Ann. of Math. (2) 73 (1961), 458-495. MR 23 \#A3997.

7. R. G. Douglas, On factoring positive operator functions, J. Math. Mech. 16 (1966), 119-126. MR 35 \#782.

8. L. Gårding and L. Hörmander, Strongly subharmonic functions, Math. Scand. 15 (1964), 93-96. MR 31 \#3621.

9. I. C. Gohberg, The factorization problem for operator functions, Izv. Akad. Nauk SSSR Ser. Mat. 28 (1964), 1055-1082; English transl., Amer. Math. Soc. Transl. (2) 49 (1966), 130-161. MR 30 \#5182.

10. H. Helson, Lectures on invariant subspaces, Academic Press, New York, 1964. MR $30 \# 1409$.

11. H. Helson and D. Lowdenslager, Prediction theory and Fourier series in several variables, Acta Math. 99 (1958), 165-202; II, ibid. 106 (1961), 175-213. MR 20 \#4155; MR 31 \#562.

12. K. Hoffman, Banach spaces of analytic functions, Prentice-Hall, Series in Modern Analysis, Prentice-Hall, Englewood Cliffs, N. J., 1962. MR 24 \#A2844.

13. M. G. KreYn, A contribution to the theory of entire functions of exponential type, Izv. Akad. Nauk SSSR 11 (1947), 309-326. (Russian) MR 9, 179.

14. T. L. Kriete, A generalized Paley-Wiener theorem, J. Math. Anal. Appl. (to appear).

15. P. D. Lax, Translation invariant spaces, Proc. Internat. Sympos. on Linear Spaces (Jerusalem, 1960), Jerusalem Academic Press, Jerusalem; Pergamon Press, Oxford, 1961. MR 25 \#4345.

16. B. Ja. Levin, Distribution of zeros of entire functions, GITTL, Moscow, 1956; English transl., Transl. Math. Monographs, vol. 5, Amer. Math. Soc., Providence, R. I., 1964. MR 19, 402; MR 28 \#217. 
17. D. Lowdenslager, On factoring matrix valued functions, Ann. of Math. (2) 78 (1963), 450-454. MR 27 \#6094.

18. P. Masani, Shift invariant spaces and prediction theory, Acta Math. 107 (1962), 275-290. MR 25 \#4344.

19. B. Moore, Outer factorization for vectorial Toeplitz operators, Dissertation, University of Virginia, Charlottesville, Va., 1969.

20. —- The Szegö infimum, Proc. Amer. Math. Soc. 29 (1971), (to appear).

21. B. Sz.-Nagy and C. Foiaş, Analyse harmonique des operateurs de l'espace de Hilbert, Masson, Paris; Akad. Kiadó, Budapest, 1967. MR 37 \#877.

22. I. I. Privalov, Boundary properties of analytic functions, GITTL, Moscow, 1950; German transl., Hochschulbücher für Math., Band 25, VEB Deutscher Verlag, Berlin, 1956. MR 13, 926; MR 18, 727.

23. E. A. Robinson, Extremal representation of stationary stochastic processes, Ark. Mat. 4 (1962), 379-384. MR 25 \#4576.

24. M. Rosenblatt, A multi-dimensional prediction problem, Ark. Mat. 3 (1958), 407-424. MR 19, 1098.

25. M. Rosenblum, Vectorial Toeplitz operators and the Fejêr-Riesz theorem, J. Math. Anal. Appl. 23 (1968), 139-147. MR 37 \#3378.

26. M. Rosenblum and J. Rovnyak, Factorization of operator valued entire functions, Indiana Univ. Math. J. 20 (1970), 157-173.

27. Ju A. Rozanov, Stationary random processes, Fizmatgiz, Moscow, 1963; English transl., Holden-Day, San Francisco, Calif., 1967. MR 28 \#2580; MR 35 \#4985.

28. N. Wiener and P. Masani, The prediction theory of multivariate stochastic processes. I. The regularity condition, Acta Math. 98 (1957), 111-150; II. The linear predictor, ibid., 99 (1958), 93-137. MR 20 \#4323; MR 20 \#4325.

University of Virginia, Charlottesville, Virginia 22901 